\title{
The Reciprocity Gap Functional for Identifying Defects and Cracks
}

\author{
H.D. Bui, A. Constantinescu and H. Maigre \\ Laboratoire de Mécanique des Solides, CNRS, \\ Ecole Polytechnique, Palaiseau, France
}

\begin{abstract}
The recovery of defects and cracks in solids using overdetermined boundary data, both the Dirichlet and the Neumann types, is considered in this paper. A review of the method for solving these inverse problems is given, focusing particularly on linearized inverse problems. It is shown how the reciprocity gap functional can solve nonlinear inverse problems involving identification of cracks and distributed defects in bounded solids. Exact solutions for planar cracks in 3D solids are given for static elasticity, heat diffusion and transient acoustics.
\end{abstract}

\section{Introduction and Scope}

There are many classes of inverse problems for detection of defects in solids: recovery of distributed coefficients, densities, identification of cavities and cracks.

The recovery of coefficients or densities in a domain from line integrals, known as "Ray Tomography", with X-rays or Gamma-rays, single or double photon emission... is possible owing, on the one hand, to highly sophisticated data acquisition systems such as Scanners, Photonic Collimators and, on the other hand, to mathematical techniques like the Radon transform and its exact inverse, with or without absorption. The case of Gamma ray tomography, taking account of the Compton electron diffusion, has been investigated recently by Nguyen and Truong (2002) and Nguyen et al (2002) who gave the exact inverse of the socalled "Conical Radon Transform". For optical absorption and scattering tomography, the 
mathematical models are given by the Boltzmann transport equation and the diffusion equation. For a review of the topic, see Arridge (1999).

Applications of acoustics in geophysics and non destructive testing of materials are based on mechanical set-ups like the transducers which collect data on their boundary. Mathematically one deals here with inverse scattering theories which are based on some approximations: incident plane waves, Bom's approximation, Kirchhoff's approximation (see Bui, 1993, 1994). Analytical solutions exist only for some specific problems. For example, in "Acoustics tomography", under the above approximations, an exact solution to the inverse scattering of a rigid inclusion has been given by Bojarski (1981). The classical "Elastic Wave Tomography" using the Kirchhoff approximation, the far-field analysis and the assumption of smallness of the defects, leads to the so-called POFFIS method (Physical Optics Far Field Inverse Scattering). Most works applied to Geophysics are generally based on numerical methods such as Finite Elements Method, Boundary Integral Equations, Optimal Control theory ( Lions, 1971).

Recently a large amount of work has been devoted to "Generalized Tomography" applied to different physical phenomena ranging from elliptic to parabolic and hyperbolic equations. Such works are yet at the stage of primary developments of methods for computational and for pure and applied mathematical purposes. Despite the existence of new devices such as the Infra-Red camera already used for investigation of delaminations in composites structures or functional observations in Biology, its industrial applications are not well developped. One explanation is the lack of exact solutions to thermal inverse problems which can be used as a benchmark for computational solutions and, above all, the difficulties encountered in numerical methods for ill-posed inverse problems.

The aim of this paper is to review some recent works done in the applied mathematics for identifying defects in solids. It focuses mainly on the following topics:

a. Derivation of the "Observation Equation" using the notion of "Defect Indicators".

b. General considerations on non-linear and linearized inverse problems.

c. Linear inverse problems settings; Ill-posedness; Regularizations methods; Mathematical aspects; The Cauchy Problem.

d. Exact solutions of the inverse problems for identifying a planar crack in the 3D case, for elliptic, parabolic and hyperbolic equations, from overdetermined boundary data.

\section{Defects and cracks indicators}

The boundary data for recovering the defects provides qualitative and quantitative informations about the unknowns by means of defect indicators. If there exists a relationship between the boundary data and the parameters which characterizes the unknown defects, this relationship can be used as a defect indicator. Generally the indicator is a boundary integral which vanishes in the case of absence of defect or relates to the strength of the singularities inside the body, the location of point forces, or the crack geometry, etc.

The method for obtaining indicators are based on conservation laws which transfer the information about the mechanical field inside the body to the exterior boundary $\mathrm{S}$ where boundary data can be obtained, without solving any boundary value problem. Let us consider 
some types of indicators in the following examples. Consider a first class of indicator defined by a conservation law of the form $-\operatorname{div}(\mathbf{A})=\mathrm{B}$ in $\Omega$, with $\mathrm{B}$ a source term.

The defect indicator is given by the boundary integral

$$
I=-\int_{S} \text { A.n dS }
$$

over the exterior surface $S$ of the body. The integral (1) vanishes (or not) if $B=0$ (or $B \neq 0$ ), indicating the abscence (or presence) of the source. As an example, consider the stress field $\sigma(\mathbf{x})$ in a $3 \mathrm{D}$ solid,

$$
-\operatorname{div} \sigma(\mathbf{x})=\mathbf{f} \delta(\mathbf{x}-\mathbf{a}) \text { in } \Omega
$$

with the point force $f \delta(\mathbf{x}-\mathbf{a})$. The integral (1) is equal to the total force $\mathbf{f}$.

Consider another class of indicator more general than (1), in the case of linear elasticity under small strain. Denote by $\sigma[\mathbf{u}](\mathbf{x})$ the stress field corresponding to the displacement field $\mathbf{u}(\mathbf{x})$ in the solid due to the point force $\mathbf{f} \delta(\mathbf{x}-\mathbf{a})$. Introduce an auxiliary field $\sigma[\mathbf{v}](\mathbf{x})$ associated to the displacement field $\mathbf{v}(\mathbf{x})$, without body force. We get

$$
-\operatorname{div}\{\sigma[\mathbf{u}] . \mathbf{v}-\sigma[\mathbf{v}] . \mathbf{u}\}=\mathbf{v} . \mathbf{f} \delta(\mathbf{x}-\mathbf{a}) \text { in } \Omega
$$

The indicator derived from (3) is the linear form $\mathbf{v} \rightarrow \mathrm{I}(\mathbf{v})$

$$
\mathrm{I}(\mathbf{v})=-\int_{S}\{\mathbf{n} \cdot \sigma[\mathbf{u}] . \mathbf{v}-\mathbf{n} \cdot \sigma[\mathbf{v}] \cdot \mathbf{u}\} \mathrm{dS}
$$

which contains the information on both the force $\mathbf{f}$ and the point $\mathbf{a}$.

This indicator is twice the bilinear symplectic form corresponding to the energy release rate in linear Fracture Mechanics.

Exercices. Take $\mathbf{v}=\left(v_{j}\right), v_{j}=\delta_{i j}$ and verify that $I\left(v_{j}\right)=f_{i}$.

Take $\mathbf{v}=\left(v_{j}\right), v_{j}=x_{k} \delta_{i j}$ and verify that $I\left(v_{j}\right)=a_{k} f_{i} \cdot \bullet$

\subsection{Crack indicator}

Let us introduce another class of indicators more suitable for crack detection. We assume that the stress field $\sigma[\mathbf{u}](\mathbf{x})$ corresponds to the stress field of the previous 3D elastic solid with an unique intermal crack $\Sigma$, with some boundary conditions on $\mathrm{S}$ and without body force. We assume that $\mathbf{v}(\mathbf{x})$ corresponds to the auxiliary (or adjoint) field of the uncracked solid, with the same elastic constants and some different boundary conditions. The indicator given by (4) is called here the Reciprocity gap R(v)

$$
\mathrm{R}(\mathbf{v})=\int_{S}\{\mathbf{n} . \sigma[\mathbf{v}] \cdot \mathbf{u}-\mathbf{n} . \sigma[\mathbf{u}] . \mathbf{v}\} \mathrm{dS}
$$


The reason of the word "gap" in the name comes from the fact that in an uncracked body, the Betti reciprocity relation yields $R(\mathbf{v})=0$, for any $\mathbf{v}$ satisfying the elasticity equation. Hence a non vanishing value of $R(v)$ indicates the presence of a crack. More precisely by introducing the displacement jump defined as $[[\mathbf{u}]]=\mathbf{u}^{+}-\mathbf{u}^{-}$one gets

$$
\mathrm{R}(\mathbf{v})=\int_{\Sigma} \mathbf{n} \cdot \sigma[\mathbf{v}] \cdot[[\mathbf{u}]] \mathrm{d} S
$$

where $\mathbf{n}=\mathbf{n}^{-}=-\mathbf{n}^{+}$. From (5) and (6) one obtains the "observation equation"

$$
\int_{\Sigma} \mathbf{n} \cdot \sigma[\mathbf{v}] \cdot[[\mathbf{u}]] \mathrm{d} S=\int_{S}\{\mathbf{n} \cdot \sigma[\mathbf{v}] \cdot \mathbf{u}-\mathbf{n} \cdot \sigma[\mathbf{u}] \cdot \mathbf{v}\} \mathrm{d} S
$$

Equation (7) has been derived in many works as a tool for crack identification. It can be generalized to transient problems in elasticity, acoustics, and also to heat diffusion problems (See Section 7.2). The use of (7) for crack identification using an overdetermined boundary data pair ( $(\mathbf{u}, \mathbf{T}:=\mathbf{n} . \sigma[\mathbf{u}])$ will be discussed in Section 7. Let us remark that Equation (7) alone is not sufficient for identifying the crack $\Sigma$. We shall discuss further two important questions : the number of data pairs $(\mathbf{u}, \mathbf{T})$ and the nature of adjoint fields which are necessary and sufficient for a complete identification. The identifiability question is important because it is related to uniqueness of the solution of the inverse problem for exact data pair. In contrast with the identifiability problem, practical solutions to crack or defect inverse problems are based on numerical computations which generally make use of noise-contamined data pairs. In this case, the key issues are the continuity of approximate solutions with respect to data variation and an error estimate with respect to the exact solution. These questions will be examined for linear inverse problems which are simpler than nonlinear inverse problems.

\subsection{Distributed Defect Indicator}

The following example is given by Calderon (1980) for transient as well as for static heat diffusion equation. Let us restrict to the static case. Distributed defects in solids are due for example to the presence of microscopic voids and micro-cracks which change macroscopically the thermal diffusion coef ficient, from the known constant value $\mathrm{k}_{\mathrm{O}}$ to $\mathrm{k}(\mathbf{x})=\mathrm{k}_{\mathrm{O}}+\mathrm{c}(\mathbf{x})$.

Without lost of generality, we consider normalized constant so that $k_{0}=1$ and assume that $c(x)=0$ on $\partial \Omega$. In the macroscopic scale, $c(x)$ can be considered as the damage field.

The inverse problem consists of finding $\mathrm{c}(\mathbf{x})$ from measurements of surface temperature $\theta$ and temperature gradient $\partial_{\mathrm{n}} \theta$.

The equations are the following ones.

-Actual field $\theta$ (damaged solid) :

$$
\begin{array}{ll}
-\operatorname{div}\{(1+\mathrm{c}) \operatorname{grad} \theta\}=0 & \text { (in } \Omega) \\
\partial_{\mathrm{n}} \theta(\mathbf{x})=\mathrm{f}(\mathbf{x}) & (\text { on } \partial \Omega)
\end{array}
$$


-Adjoint field $\psi$ (undamaged solid):

$$
\begin{array}{ll}
- \text { divgrad } \psi=0 & \text { (in } \Omega) \\
\partial_{\mathrm{n}} \psi(\mathbf{x})=\mathrm{g}(\mathbf{x}) & (\text { on } \partial \Omega)
\end{array}
$$

The observation equation is given by :

$$
\int_{\Omega} \mathrm{c} \operatorname{grad} \theta[\mathrm{c}] \cdot \operatorname{grad} \psi \mathrm{dV}=\int_{\partial \Omega}\left\{\psi \partial_{\mathrm{n}} \theta-\theta \partial_{\mathrm{n}} \psi\right) \mathrm{dS} \quad(\equiv \mathrm{d})
$$

The explicit dependence of $\theta[c]$ on $c$ in Equation (12) and the solution of (8) and (9) under the compatibility condition $\square \int_{\partial \Omega} \mathrm{fdS}=0$ clearly show that the observation equation is nonlinear with respect to $\mathrm{c}$.

The observation equation (12) alone does not allow the determination of the scalar field $\mathrm{c}(\mathbf{x})$ since its right hand side is a scalar constant denoted by $\mathrm{d}$, for a given surface data pair $\left(\theta, \partial_{\mathrm{n}} \theta\right)$ and for a given adjoint field $\psi(\mathbf{x})$. It is necessary to establish an one-to-one mapping between $\mathrm{c}(\mathbf{x})$ and a larger family of data $\mathrm{d}(\zeta)$ depending on some family of boundary data or of adjoint fields indexed by some $\mathrm{N}$-dimensional parameter $\zeta$.

To illustrate the necessary condition, suppose for example that $\mathrm{c}(\mathbf{x})$ is a constant $\mathrm{c}$ inside a $2 \mathrm{D}$ ellipsoidal inclusion, and is equal to zero outside the inclusion, Figure 1 . To determine the constant $\mathrm{c}$ and the ellipsoidal geometry, e.g. 6 unknown parameters (the constant c, 2 coordinates of the center, the orientation of the main axis, the semi axes a and b), many procedures can be used. One can consider a single data pair $\left(\theta, \partial_{n} \theta\right)$ measured on $\partial \Omega$ and six adjoint fields, or two data pairs $\left(\theta^{1}, \partial_{n} \theta^{1}\right),\left(\theta^{2}, \partial_{n} \theta^{2}\right)$ and three adjoint fields etc. These conditions are necessary but not sufficient for solving the nonlinear inverse problem.

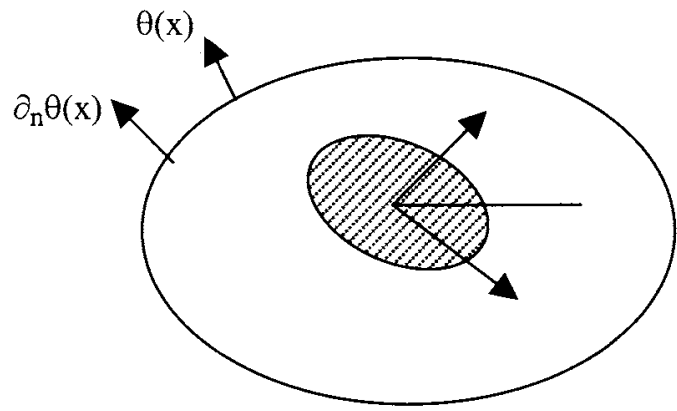

Figure 1. Two dimensional ellipsoidal inclusion. 
Calderon (1980) proposed a linearization of (12) in order to obtain a simpler inverse problem, which can be solved exactly for 3D defects. His basic idea is the consideration of a 3dimensional family of boundary data $f_{z}(\mathbf{x})$ and $g_{z}(\mathbf{x})$, with $\mathbf{z}$ being a $3 D$ vector.

The linearized inverse problem is based on the first order approximation of (12), considering $\mathrm{c}$ as a small perturbation. The linearized observation equation for the first order solution $\mathrm{c}^{\sim}$ is deduced from (12) by leaving the right hand side of (12) unchanged and changing only the left hand side as

$$
\int_{\Omega} \mathrm{c}^{\sim} \operatorname{grad} \phi \cdot \operatorname{grad} \psi \mathrm{dV}=\int_{\partial \Omega}(\psi \mathrm{f}-\theta \mathrm{g}) \mathrm{dS}
$$

where $\phi$ is the solution of the boundary value problem on the solid without defect :

$$
\begin{aligned}
& -\operatorname{div}\{\operatorname{grad} \phi\}=0 \quad \text { (in } \Omega) \\
& \partial_{\mathrm{n}} \phi(\mathrm{x})=\mathrm{f}(\mathbf{x}) \quad(\text { on } \partial \Omega)
\end{aligned}
$$

By taking a family of boundary data $f_{z}(\mathbf{x})$ and $g_{z}(\mathbf{x}), \mathbf{z} \in \mathrm{R}^{3}$, the right hand side of (13) becomes a scalar field $\mathrm{d}(\mathbf{z})$ and equation (13) becomes a Fredholm integral equation of the first kind for $\mathbf{c}^{\sim}(\mathbf{x})$ with kemel $\mathrm{K}(\mathbf{x}, \mathbf{z})=\operatorname{grad}_{\mathrm{x}} \phi(\mathbf{x} ; \mathbf{z}) \cdot \operatorname{grad}_{\mathrm{x}} \psi(\mathbf{x} ; \mathbf{z})$. As expected, the linear inverse problem for $\mathrm{c}^{\sim}$ is ill-posed because it is governed by a Fredholm integral equation of the first kind mapping the $\mathrm{x}$-space onto the z-space.

An attempt to solve the nonlinear equation has been given in (Isaacson and Isaacson, 1989) for determining $\mathrm{c}(\mathbf{x})$ within a circular inclusion (a priori knowledge on the shape). A surprising result was found for the supporting function supp[c] which takes the values 0 or 1 , depending on whether $\mathrm{c}(\mathbf{x})=0$ or $\mathrm{c}(\mathbf{x}) \neq 0$. It is found that the support functions of $\mathrm{c}(\mathbf{x})$ for nonlinear theory and $\mathbf{c}^{\sim}(\mathbf{x})$ for linearized theory are practically the same. The result is very interesting for applications because it justifies the use of the linearized theory, even when $c(x)$ is not small.

A recent study (Bui and Constantinescu, 2000) on the detection of damage in elasticity using integral equation techniques gives a similar result. Far from the inclusion and the boundary, where $\mathbf{c}(\mathbf{x})$ is assumed to be zero, the difference in the stress components between nonlinear and linearized theories is found to be negligible.

In the next section, we reconsider the comparison between nonlinear and linerarized theories for the stationary heat equation.

\section{Nonlinear and Linear Theories}

Consider the inverse problem for determining an internal perturbation $\mathrm{c}(\mathbf{x})$, using the boundary data $\theta(\mathbf{x})=\mathrm{h}(\mathbf{x})$ and $\partial_{\mathrm{n}} \theta(\mathbf{x})=\mathrm{f}(\mathbf{x})$. Instead of comparing $\mathrm{c}(\mathbf{x})$ with $\mathrm{c}^{\sim}(\mathbf{x})$, we compare the nonlinear solution $\theta^{\mathrm{NL}}(\mathbf{x})$ with the thermal field $\theta^{\mathrm{L}}(\mathbf{x})$ associated with the linear theory. We assume

i) that the perturbation is continuous and admits a series expansion in the form $c=\varepsilon c_{1}+\varepsilon^{2} c_{2}+\ldots$ with small $\varepsilon$ and,

ii) that the supports $Z_{i}$ of the functions $c_{i}(x)$ are compact and that $c_{i}(x)=0$ on $\partial Z_{i}$ and outside $Z_{i}$. The support $Z$ of $c(x)$ is given by the union of the $Z_{i}$ 's. A well-known example of such compact support functions is given by the test functions in finite element methods. 
The field $\theta(\mathbf{x})$ satisfies the Dirichlet boundary value problem:

$$
\begin{array}{cc}
-\operatorname{div}\{(1+\mathrm{c}) \operatorname{grad} \theta\}=0 \quad \text { (in } \Omega) \\
\theta(\mathbf{x})=\mathrm{h}(\mathbf{x}) \quad(\text { on } \partial \Omega)
\end{array}
$$

and admits the series expansion $\theta(\mathbf{x})=\theta_{0}+\varepsilon \theta_{1}(\mathbf{x})+\varepsilon^{2} \theta_{2}(\mathbf{x})+\ldots$ The nonlinear thermal field $\theta^{N L}(\mathbf{x}) \equiv \theta(\mathbf{x})$ admits the integral representation $(\partial \theta / \partial \mathrm{n} \equiv \mathrm{f}(\mathbf{y}))$

$$
\theta(\mathbf{x})=\int_{\partial \Omega}\left\{\mathrm{G}(\mathbf{x}, \mathbf{y}) \mathrm{f}(\mathbf{y})-\mathrm{h}(\mathbf{y}) \partial_{\mathrm{n}_{\mathbf{y}}} \mathrm{G}(\mathbf{x}, \mathbf{y})\right\} \mathrm{dS}_{\mathbf{y}}+\int_{\mathrm{Z}} \mathrm{G}(\mathbf{x}, \mathbf{y}) \operatorname{div}\{\mathrm{c}(\mathbf{y}) \operatorname{grad} \theta(\mathbf{y})\} \mathrm{dV} \mathrm{y}_{\mathrm{y}}
$$

where $G(\mathbf{x}, \mathbf{y})$ is the Green's function. We call $\theta_{0}(\mathbf{x})$ the zero-order solution satisfying the equations:

$$
\begin{array}{lc}
-\operatorname{div}\left\{\operatorname{grad} \theta_{0}\right\}=0 & (\text { in } \Omega) \\
\theta_{0}(\mathbf{x})=\mathrm{h}(\mathbf{x}) & (\text { on } \partial \Omega)
\end{array}
$$

The zero order term $\theta_{0}(\mathbf{x})$ admits the integral representation

$$
\theta_{0}(\mathbf{x})=\int_{\partial \Omega}\left\{\mathrm{G}(\mathbf{x}, \mathbf{y}) \partial_{\mathrm{n}_{\mathrm{y}}} \theta_{0}(\mathbf{y})-\mathrm{h}(\mathbf{y}) \partial_{\mathrm{n}_{\mathrm{y}}} \mathrm{G}(\mathbf{x}, \mathbf{y})\right\} \mathrm{dS} \mathrm{S}_{\mathbf{y}}
$$

In what follows, the sum of the first two terms will be refered to as the linear solution

$$
\theta L(\mathbf{x})=\theta_{0}(\mathbf{x})+\varepsilon \theta_{1}(\mathbf{x})
$$

By substituting the series expansions of $\mathrm{c}(\mathbf{x})$ and $\theta(\mathbf{x})$ in (16) and (17) and considering the term $O(\varepsilon)$, we obtain the governing equations for $\theta_{1}(x)$

$$
\begin{array}{lr}
-\operatorname{div}\left\{\operatorname{grad} \theta_{1}\right\}=\operatorname{div}\left\{\mathrm{c}_{1}(\mathbf{x}) \operatorname{grad} \theta_{0}(\mathbf{x})\right\} & \text { (in } \Omega) \\
\theta_{1}(\mathbf{x})=0 & (\text { on } \partial \Omega)
\end{array}
$$

The field $\theta_{1}(\mathbf{x})$ admits the integral representation

$$
\theta_{1}(\mathbf{x})=\int_{\partial \Omega} \mathrm{G}(\mathbf{x}, \mathbf{y}) \partial_{\mathrm{n}_{\mathrm{y}}} \theta_{1}(\mathbf{y}) \mathrm{dS} \mathbf{S}_{\mathrm{y}}+\int_{\mathrm{Z}_{1}} \mathrm{G}(\mathbf{x}, \mathbf{y}) \operatorname{div}\left\{\mathbf{c}_{1}(\mathbf{y}) \operatorname{grad} \theta_{0}(\mathbf{y})\right\} \mathrm{dV} \mathrm{V}_{\mathrm{y}}
$$

Using the boundary condition $c_{1}(y)=0$ on $\partial Z_{1}$, it can be shown that the difference $\Delta$ between the nonlinear solution and the first order solution is given by

$$
\begin{gathered}
\Delta=\theta^{N L}(\mathbf{x})-\theta^{L}(\mathbf{x})=\int_{\partial \Omega} \mathrm{G}(\mathbf{x}, \mathbf{y})\left\{\partial_{\mathrm{n}_{\mathrm{y}}} \theta-\partial_{\mathrm{n}_{\mathrm{y}}} \theta_{0}-\varepsilon \partial_{\mathrm{n}_{\mathrm{y}}} \theta_{1}\right\} \mathrm{dS} \mathrm{y}_{\mathrm{y}} \\
-\varepsilon^{2} \int_{\mathrm{Z}_{1}} \operatorname{grad}_{\mathbf{x}} \mathrm{G}(\mathbf{x}, \mathbf{y}) \mathbf{c}_{1}(\mathbf{y}) \operatorname{grad}_{\mathrm{y}} \theta_{1}(\mathbf{y}) \mathrm{dV}_{\mathrm{y}}
\end{gathered}
$$


Both terms of the right hand side of (25) are of order $\mathrm{O}\left(\varepsilon^{2}\right)$. By differentiating (25), we obtain the difference between their gradients $\Gamma=\operatorname{grad}_{\mathrm{x}} \Delta$

$$
\begin{gathered}
\Gamma(\mathbf{x})=\int_{\partial \Omega} \operatorname{grad}_{\mathrm{x}} \mathrm{G}(\mathbf{x}, \mathbf{y})\left\{\partial_{\mathrm{n}_{\mathrm{y}}} \theta-\partial_{\mathrm{n}_{\mathrm{y}}} \theta_{0}-\varepsilon \partial_{\mathrm{n}_{\mathrm{y}}} \theta_{1}\right\} \mathrm{dS}_{\mathrm{y}} \\
-(\mathrm{fp}) \varepsilon^{2} \int_{\mathrm{Z}_{1}} \operatorname{grad}_{\mathrm{x}} \operatorname{grad}_{\mathrm{y}} \mathrm{G}(\mathbf{x}, \mathbf{y}) \mathrm{c}_{1}(\mathbf{y}) \operatorname{grad}_{\mathrm{y}} \theta_{1}(\mathbf{y}) \mathrm{dV} \mathrm{V}_{\mathrm{y}} \quad \mathbf{x} \notin\left(\mathrm{Z}_{1} \cup \partial \Omega\right)
\end{gathered}
$$

where (fp) stands for the Hadamard finite part sense. A close inspection of (26) shows that the difference between the thermal gradient is significant only inside or near $\mathrm{Z} \supset \mathrm{Z}_{1}$ because of the following reasons :

1. the kemel of the first term behaves as $\left|\operatorname{grad}_{x} G\right| \approx|\mathbf{x}-\mathbf{y}|^{-2}$, with $|\mathbf{x}-\mathbf{y}|=\operatorname{distance}(\mathbf{x}, \partial \Omega)$.

Therefore the first integral term is significant only in the vicinity of $\partial \Omega$, due to the difference in normal derivatives $\partial_{\mathrm{n}_{\mathrm{y}}} \theta-\partial_{\mathrm{n}_{\mathrm{y}}}\left(\theta_{0}+\varepsilon \theta_{1}\right) \approx \mathrm{O}\left(\varepsilon^{2}\right)$. Note that in the derivation of the approximate solution, $\mathbf{c}^{\sim}(\mathbf{x})$, it is assumed that the same normal derivative holds for $\theta^{\mathrm{L}}(\mathbf{x})$ and $\theta(\mathbf{x})$ so that $\left\{\partial_{\mathrm{n}_{\mathrm{y}}} \theta-\partial_{\mathrm{n}_{\mathrm{y}}}\left(\theta_{0}+\varepsilon \theta_{1}\right)\right\} \approx 0$.

2 . the second term, understood in the Hadamard finite-part sense, decreases as $\left|\operatorname{grad}_{\mathrm{x}} \operatorname{grad}_{\mathrm{y}} \mathrm{G}\right| \approx|\mathbf{x}-\mathbf{y}|^{-3}$, as the distance $\mathrm{r}=\operatorname{distance}\left(\mathbf{x}, \mathrm{Z}_{1}\right)$ increases.

The difference $\Delta$ between nonlinear and linear thermal fields decreases as the distance to $Z_{1}$ increases, and the decrease of $\Gamma$ is much more rapid.

These analyses show that far away from $Z_{1}$ we find that the thermal fields $\theta^{N L}(\mathbf{x}), \theta^{L}(\mathbf{x})$ and their respective gradients $\operatorname{grad}_{x} \theta^{N L}(\mathbf{x})$, $\operatorname{grad}_{x} \theta^{L}(\mathbf{x})$, are quite similar. In other words, the perturbation of the thermal coefficient $c(x)$ is not significant outside $Z_{1}$, while by assumption, the perturbation $\varepsilon c_{1}(\mathbf{x})$ vanishes outside $Z_{1}$, consequently vanishes too outside $Z$ (which contains the subset $Z_{1}$ ).

This discussion indirectly shows that the property observed in (Isaacson and Isaacson, 1989) about the support of the nonlinear solution $\mathrm{c}(\mathbf{x})$ for circular inclusion and that of the linearized theory $\mathrm{c}^{\sim}(\mathbf{x})$, in the Calderon sense, Equation (13), seems to be likely for an arbitrary geometry of the inclusion.

Remark 1. The example of the thermal inverse problem shows that a relationship between the unknown coefficient $k(\mathbf{x})$ and the boundary data pair $d=\left\{\theta^{d}, \partial_{n} \theta d\right\}$ can be written formally as $\mathrm{A}(\mathrm{k})=\mathrm{d}$, where $\mathrm{A}$ is a nonlinear integral operator, the kemel of which is defined implicitly by equations (8) and (9). A simpler approximate observation equation is derived in discretized form, if we set $\mathrm{k}=\left\{\mathrm{k}_{1}, \mathrm{k}_{2}, \mathrm{k}_{3}, \ldots \mathrm{k}_{\mathrm{n}}\right\}$. By means of the Finite Element Method, one solves (8) and (9) using the Neumann boundary condition $\partial_{n} \theta=f\left(\right.$ or $\left.\partial_{n} \theta^{d}\right)$ and calculates the boundary temperature $\theta \mathrm{cal}\left(\mathrm{k}_{1}, \mathrm{k}_{2}, \mathrm{k}_{3}, \ldots \mathrm{k}_{\mathrm{n}}\right)$. The inverse problem is defined as the optimization of an objective function.

Generally, the least square method is used to minimize the "error" between predicted and measured temperatures

$$
\mathrm{k}=\arg \operatorname{Min}_{\mathrm{k}}\left|\theta \operatorname{cal}\left(\mathrm{k}_{1}, \mathrm{k}_{2}, \mathrm{k}_{3}, \ldots \mathrm{k}_{\mathrm{n}}\right)-\theta \mathrm{d}\right| 2
$$

The difficulty of such numerical approach lies on the evaluation of the gradient of the error functional with respect to $\mathrm{k}_{\mathrm{i}}$ which requires a great number of solutions of the equations $(8)$ and 
(9) for different increments $\delta \mathrm{k}_{\mathrm{i}}$. It can be overcame by using adjoint equations (See, e.g., Constantinescu, 1995 ).

Alternatively, instead of using the Neumann bounadry condition, one can solve the thermal equation with the Dirichlet boundary condition $\theta=\theta^{\mathrm{d}}$ and then calculate the optimal flux $\partial_{\mathrm{n}} \theta \mathrm{cal}\left(\mathrm{k}_{1}, \mathrm{k}_{2}, \mathrm{k}_{3}, \ldots \mathrm{k}_{\mathrm{n}}\right)$ which approaches the datum $\partial_{\mathrm{n}} \theta^{\mathrm{d}}$

$$
\mathrm{k}=\arg \operatorname{Min}_{\mathrm{k}}\left|\partial_{\mathrm{n}} \theta^{\mathrm{cal}}\left(\mathrm{k}_{1}, \mathrm{k}_{2}, \mathrm{k}_{3}, \ldots \mathrm{k}_{\mathrm{n}}\right)-\partial_{\mathrm{n}} \theta^{\mathrm{d}}\right|^{2}
$$

A symmetrical method consists of minimizing the "constitutive law error functional" (see Kohn and Vogelius, 1984 and Constantinescu, 1994, 1995).

$$
\mathrm{F}(\mathrm{q}, \theta, \mathrm{k})=(1 / 2) \int_{\Omega}\left(\mathrm{k}^{1 / 2} \operatorname{grad} \theta+\mathrm{q} \mathrm{k}^{-1 / 2}\right)^{2} \mathrm{dV}
$$

where the field $\theta$ satisfies the Dirichlet boundary condition $\theta=\theta^{d}$ while $q$ satisfies the equilibrium equation $\operatorname{div}(q)=0$ and the Neumann boundary condition $q=f(k=1$ on the boundary). The constitutive law

$$
\mathrm{q}+\operatorname{kgrad} \theta=0
$$

is solution of the minimization of the "polyconvex" functional $\mathrm{F}$, convex with respect to each variable separately, but not convex with respect to all together.

Remark 2. The nonlinear equation $\mathrm{A}(\mathrm{u})=\mathrm{y}, \mathrm{u} \in \mathrm{X}, \mathrm{y} \in \mathrm{Y}$ is generally solved by methods of linearization. It is assumed that the Frechet derivative $A^{\prime}(u)$ exists. The least square method leads to the minimization problem $\operatorname{Min}_{\mathrm{u}}\|\mathrm{A}(\mathrm{u})-\mathrm{y}\|^{2}$, with the norm $\|$.$\| in the \mathrm{Y}$-space. The solution is obtained formally by the multistage quadratic programming which, starting from a guess value (or a priori knowledge) $\mathrm{u}^{0}$, calculates the updated solution $\mathrm{u}^{\mathrm{k}+1}=\mathrm{u}^{\mathrm{k}+\mathrm{s}^{\mathrm{k}}}$ with

$$
\mathrm{s}^{\mathrm{k}}=\operatorname{Arg}\left(\operatorname{Min}_{\mathrm{s}}\left\|\mathrm{A}\left(\mathrm{u}^{\mathrm{k}}\right)+\mathrm{A}^{\prime}\left(\mathrm{u}^{\mathrm{k}}\right) \mathrm{s}-\mathrm{y}\right\|^{2}\right), \quad \mathrm{s} \in \mathrm{X}
$$

Each stage of the calculation of $\mathrm{s}^{\mathrm{k}}$ corresponds to a linear inverse problem $\mathrm{A}^{\prime}\left(\mathrm{u}^{\mathrm{k}}\right) \mathrm{s}=\mathrm{d}$, with $\mathrm{d}=\mathrm{y}-\mathrm{A}(\mathrm{uk})$.

\section{Linear Inverse Problems Settings in Hilbert Spaces}

Let $\mathrm{X}$ be the set of unknown model parameters and $\mathrm{Y}$ be the set of observable or accessible data used for recovering unknown parameters. By model parameters we include unknown mechanical fields, unknown geometry, material constants etc.

Consider two Hilbert spaces $\left(\mathrm{X},(., .)_{\mathrm{X}},\|\|.\right)$ and $\left(\mathrm{Y},(., .)_{\mathrm{Y}},||.\right)$ with inner products $(., .)_{\mathrm{X}}$, $(., .)_{Y}$, and respective associated norms $\|\cdot\|,|\cdot|$. We consider a continuous linear mapping A from $\mathrm{X}$ to $\mathrm{Y}$, which arises in many inverse problems or in the linearisation procedure of nonlinear problems. We assume that the map $A$ and its adjoint $A^{*}$ are bounded. For given 
datum $\mathrm{d} \in \mathrm{Y}$, one considers the problem of finding the solution of the so-called « observation equation $»$ :

$$
\mathrm{Au}=\mathrm{d}, \quad \mathrm{u} \in \mathrm{X}, \quad \mathrm{d} \in \mathrm{Y}
$$

One important difference with usual direct problems is that inverse problems are generally ill-posed. This means that $\mathrm{A}$ is not always invertible, or solutions do not always exist, and in the case of existence of solutions, these solutions do not depend continuously on the data. The sensitivity of the solution to errors in data is the very common feature of ill-posed problems.

It is necessary to introduce a «regularization » process in order to obtain a well-posed problem, which is required to be "close " to the initial one. These two objectives - good mathematical property in the regularization process and physically acceptable model - are often so contradictory that, according to P. Sabatier (1987), their satisfaction may be rather a matter of "Art » than "Sciences ».

The equation (27) can be writen in the variational form

$$
(A u, A v)_{Y}=(d, A v)_{Y}, u \in X, d \in Y, \forall v \in X
$$

does not have an unique solution, because the bilinear form $(\mathrm{Au}, \mathrm{Av})_{\mathrm{Y}}$ is not assumed coercive. Regularization techniques, introduced in (Tikhonov and Arsenine, 1976, 1986), consist in the construction of "solutions" stable with respect to the variation of the datum $d$, by " changing " slightly the map A, or by introducing coercive variational equations.

\subsection{Coercive Variational Equation}

We introduce an additional term $(\alpha>0)$ in the variational equation (28)

$$
(\mathrm{Au}, \mathrm{Av})_{\mathrm{Y}}+\alpha(\mathrm{u}, \mathrm{v})_{\mathrm{X}}=(\mathrm{d}, \mathrm{Av})_{\mathrm{Y}}, \mathrm{u} \in \mathrm{X}, \forall \mathrm{v} \in \mathrm{X}
$$

The added term ensures the coercivity of the bilinear form which can be rewritten as

$$
\left(\left(\mathrm{A}^{*} \mathrm{~A}+\alpha \mathrm{I}\right) \mathrm{u}, \mathrm{v}\right)_{\mathrm{X}}=\left(\mathrm{A}^{*} \mathrm{~d}, \mathrm{v}\right)_{\mathrm{X}}, \mathrm{u} \in \mathrm{X}, \forall \mathrm{v} \in \mathrm{X}
$$

where $\left(A^{*} A+\alpha I\right)$ is invertible. The solution of the modified equation (29) is

$$
u(\alpha)=(A * A+\alpha I)^{-1} A * d
$$

A large constant $\alpha$ leads to unphysical solution, while a too small value of the constant $\alpha$ yields unstable numerical solutions. There exists an optimal choice of the regularizing parameter, proposed by Kitagawa (1987). But this optimal choice is not essential for the following discussions. It is important to observe that if the solution $u$ of (28) exists, the solution is unique only when $A$ is strictly positive $A>0$ and that the solution $u(\alpha)$ in (31) with the same datum $d$ tends towards the exact solution $u$ in $X,\|u(\alpha)-u\| \rightarrow$, as $\alpha \rightarrow 0$. However, in 
most inverse problems for identifying defects and cracks, the condition $A>0$ is not always satisfied.

Remark. It is worth mentionning a geometrical interpretation of the Tikhonov regularization procedure. Suppose that there is an a priori knowledge about the solution of (27) given by the constraint $\left\|\mathbf{u}-\mathrm{u}_{\mathrm{o}}\right\|<\beta$ with known $\mathrm{u}_{\mathrm{o}}$ and $\beta>0$. An approximate solution of (27), with an a posteriori residual error $\varepsilon>0$, is given by any $u$ belonging to the intersection $C$ of two convexes $|A u-d|<\varepsilon$ and $\left\|\mathrm{u}-\mathrm{u}_{\mathrm{o}}\right\|<\beta$. Either the intersection $\mathrm{C}$ is void (no solution to the inverse problem exists) or not void (there is an infinite number of approximate solutions). In the latter case, one possible solution can be chosen as follows.

Let us remark that the intersection $C$ is bounded by two ellipsoids, $C_{2} \supset C_{\supset} C_{1}$, from below by the set $C_{1}$ of $u$ such that $|A u-d|^{2} / \varepsilon^{2}+\left\|u-u_{o}\right\|^{2} / \beta^{2} \leq 1$ and from above by the set $C_{2}$ of $u$ such that $|\mathrm{Au}-\mathrm{d}|^{2} / \varepsilon^{2}+\left\|\mathrm{u}-\mathrm{u}_{\mathrm{o}}\right\|^{2} / \beta^{2} \leq 2$.

A solution (not unique) satisfying the constraints $|A u-d|<\varepsilon$ and $\left\|\mathrm{u}-\mathrm{u}_{\mathrm{o}}\right\|<\beta$ is given by the common centre of $\mathrm{C}_{1}$ and $\mathrm{C}_{2}$ or by the solution of the minimisation problem

$$
\mathbf{u}^{(\alpha)}=\operatorname{Arg}_{\operatorname{Min}}\left\{|\operatorname{Au}-\mathrm{d}|^{2}+\alpha\left\|\mathrm{u}-\mathrm{u}_{\mathbf{o}}\right\|^{2}\right\}
$$

with $\alpha=\varepsilon^{2} / \beta^{2}$ which can be interpreted as the Lagrangian multiplier. The solution of the minimization problem (32) is unique and given by

$$
u^{(\alpha)}=u_{0}+\left(A^{*} A+\alpha I\right)^{-1} A^{*}\left(d-A u_{0}\right)
$$

\subsection{Continuity Property}

Mathematically, the regularized solution $u^{(\alpha)}$ depends continuously on the datum $d$. This is the consequence of the Lax-Milgram theorem, based on two properties :

1. $\mathrm{A}^{*} \mathrm{~A}+\alpha \mathrm{I}$ is bounded,

2. $\mathrm{A}^{*} \mathrm{~A}+\alpha \mathrm{I}$ is coercive.

The linear operator $A^{*} A: X \rightarrow X$ is self-adjoint and positive, but not strictly positive. For the continuity property and the convergence property as $\alpha \rightarrow 0$, see the following Exercices.

Exercice. Prove the inequality : $\left\|u^{(}(\alpha)(d)-u^{(\alpha)}\left(d^{\prime}\right)\right\| \leq \alpha^{-1}\|A\| .\left|d-d^{\prime}\right|$, for any $d, d^{\prime}$.

Proof: $\quad$ Consider two data d, d' and the corresponding solutions u, u' of (29). For convenience we write simply $\mathrm{u}=\mathrm{u}(\alpha)(\mathrm{d})$ and $\mathrm{u}^{\prime}=\mathrm{u}(\alpha)\left(\mathrm{d}^{\prime}\right)$.

By substracting the corresponding bilinear forms one gets:

$$
\begin{aligned}
& \left(\mathrm{A}\left(\mathrm{u}-\mathrm{u}^{\prime}\right), \mathrm{Av}\right)_{\mathrm{Y}}+\alpha\left(\mathrm{u}-\mathrm{u}^{\prime}, \mathrm{v}\right)_{\mathrm{X}}=\left(\mathrm{d}-\mathrm{d}^{\prime}, \mathrm{Av}\right)_{\mathrm{Y}} \quad \forall \mathrm{v} \in \mathrm{X} \\
\Rightarrow & \left(\mathrm{A}^{*} \mathrm{~A}\left(\mathrm{u}-\mathrm{u}^{\prime}\right), \mathrm{v}\right)_{\mathrm{X}}+\alpha\left(\mathrm{u}-\mathrm{u}^{\prime}, \mathrm{v}\right)_{\mathrm{X}}=\left(\mathrm{d}-\mathrm{d}^{\prime}, \mathrm{Av}\right)_{\mathrm{Y}} \quad \forall \mathrm{v} \in \mathrm{X}
\end{aligned}
$$

Take : $v=u-u^{\prime}$, one gets

$$
\begin{gathered}
\left\|\mathrm{A}^{*} \mathrm{~A}\right\| .\left\|\mathrm{u}-\mathrm{u}^{\prime}\right\|^{2}+\alpha\left\|\mathrm{u}-\mathrm{u}^{\prime}\right\|^{2} \leq\|\mathrm{A}\| .\left|\mathrm{d}-\mathrm{d}^{\prime}\right| . \mid\left\|\mathrm{u}-\mathrm{u}^{\prime}\right\| \Rightarrow \\
\Rightarrow \alpha\left\|\mathrm{u}-\mathrm{u}^{\prime}\right\|^{2} \leq\|\mathrm{A}\|\left|\mathrm{d}^{2}-\mathrm{d}^{\prime}\right| .\left|\left\|\mathrm{u}-\mathrm{u}^{\prime}\right\| \Rightarrow \alpha\left\|\mathrm{u}-\mathrm{u}^{\prime}\right\| \leq\|\mathrm{A}\| .\right| \mathrm{d}^{\prime}-\mathrm{d}^{\prime} \mid
\end{gathered}
$$

Exercice. (Convergence property). Prove that $\|u(\alpha)(d)-u(d)\| \rightarrow 0$, as $\alpha \rightarrow 0$. 


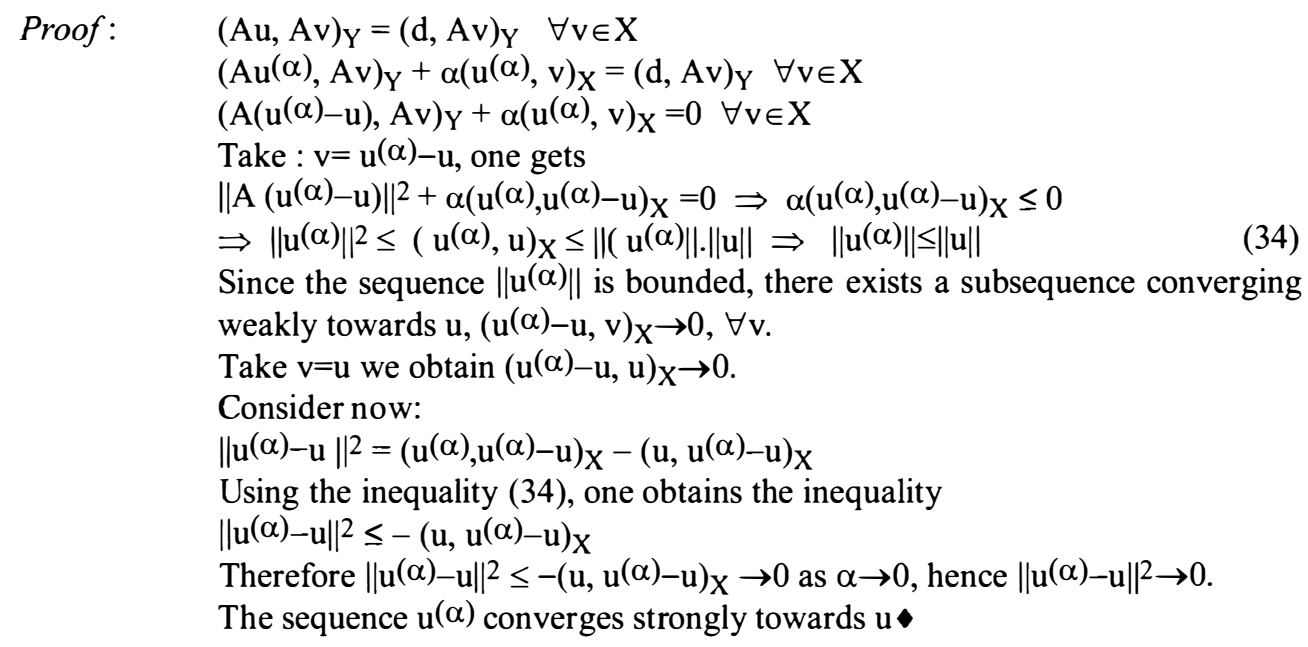

\subsection{Error Estimate}

One important point in mathematical works is to derive the error estimate with respect to the (yet unknown) exact solution $\mathrm{u}^{\mathrm{ex}}$. There are some results reported in the literature for Inverse Problem in Hilbert spaces settings. The main point is to make a comparison between the regularized solution $u^{(\alpha)}(d)$ and the exact one $u^{\text {ex }}$, even if the exact solution $u^{\text {ex }}$ is not yet known.

What is the meaning of « exact » solution?

Let us introduce some terminologies. A pair $\left\{\mathrm{u}^{\mathrm{ex}}, \mathrm{d}^{\mathrm{ex}}\right\}$ is called exact or compatible if it satisfies the observation equation

$$
A u^{e x}=d^{e x}, \quad u^{e x} \in X, \quad d^{e x} \in Y .
$$

The given data $\mathrm{d}^{\mathrm{ex}}$ may satisfy some compatibility condition for the existence of an unique exact solution $\mathrm{u}^{\mathrm{ex}}$ in $\mathrm{X}$. However it is not always possible to write explicitly the compatibility condition on $\mathrm{d}^{\mathrm{ex}}$, even if precise informations on the physical nature of the problem are known. There is of course some exception (As an example, we keep in mind the condition on the Neumann boundary data for the Laplace equation $\int \partial_{n} u d S=0$ ).

In practice, to obtain such a pair, one can take for example some $u^{\mathrm{ex}}$ and define $\mathrm{d}^{\mathrm{ex}}$ as the direct image $A u^{e x}$. Such a construction of the exact pair $\left\{u^{\mathrm{ex}}, \mathrm{d}^{\mathrm{ex}}\right\}$ is based on the solution of direct problems which gives indeed a compatible pair with respect to the inverse problem in consideration.

The knowledge of an exact pair, given by solutions of direct problems, is useful for checking the algorithm of approximate numerical solutions. One considers a model with known geometry and materials constants and known boundary conditions data for calculating $\left\{\mathrm{u}^{\mathrm{ex}}, \mathrm{d}^{\mathrm{ex}}\right\}$. Then one makes use of the numerical data $\mathrm{d}^{\mathrm{ex}}$ for recovering the approximate numerical solution $\mathrm{u}(\alpha)$ of the inverse problem. 
Another constructive solution from given data $\mathrm{d}^{\mathrm{ex}}$, consists of establishing explicit formulae giving the solution $u^{e x}$. Exact solutions given in Section 7 fall in this category of constructive solutions.

The main result in error estimate is stated in the following theorem.

Suppose that there exists $d^{1}$ such that $u^{e x}=A^{*} d^{l}$. Then, the error $\left\|u^{(\alpha)}-u^{e x}\right\|$ is given by

$$
|| \mathbf{u}^{(\alpha)}-\mathrm{u}^{\mathrm{ex}}||<(1 / 2) \alpha^{-1 / 2}\left(\left|\mathrm{~d}-\mathrm{d}^{\mathrm{ex}}\right|+\alpha\left|\mathrm{d}^{1}\right|\right)
$$

The proof is given in the next Exercice.

The estimate (35) depends on both the regularisation parameter $\alpha$ which introduces some error on the modelling and the error on the datum $\left|d-d^{e x}\right|$ in comparison with the compatible one. One gets the best possible error estimate by taking the regularization parameter proportional to the datum error $\alpha=\mathrm{k} \varepsilon$

$$
\left|\mathrm{d}-\mathrm{d}^{\mathrm{ex}}\right|<\varepsilon \quad \Rightarrow \quad\left\|\mathrm{u}^{(\alpha)}-\mathrm{u}^{\mathrm{ex}}\right\|<(1 / 2) \mathrm{k}^{-1 / 2} \varepsilon^{1 / 2}\left(1+\mathrm{k}\left|\mathrm{d}^{\mathrm{l}}\right|\right)
$$

Exercice. Suppose that there exists $d^{1}$ such that $u^{e x}=A^{*} d^{1}$, prove the estimate (35).

Proof: $\quad$ The exact pair $\left(\mathrm{u}^{\mathrm{ex}}, \mathrm{d}^{\mathrm{ex}}\right)$ satisfies the variational equation

$$
\left(A u^{\mathrm{ex}}, A v\right)_{\mathrm{Y}}=\left(\mathrm{d}^{\mathrm{ex}}, \mathrm{Av}\right)_{\mathrm{Y}} \forall \mathrm{v} \in \mathrm{X} \text {, }
$$

The approximate solution $\left(\mathrm{u}^{(\alpha)}, \mathrm{d}\right)$ satisfies the equation

$\left(\mathrm{Au} \mathrm{u}^{(\alpha)}, \mathrm{Av}\right)_{\mathrm{Y}}+\alpha\left(\mathrm{u}^{(\alpha)}, \mathrm{v}\right)_{\mathrm{X}}=(\mathrm{d}, \mathrm{Av})_{\mathrm{Y}} \forall \mathrm{v} \in \mathrm{X}$

which can be written as :

$\left(\mathrm{Au} u^{(\alpha)}, \mathrm{Av}\right)_{\mathrm{Y}}+\alpha\left(\mathrm{u}^{(\alpha)}-\mathrm{u}^{\mathrm{ex}}, \mathrm{v}\right)_{\mathrm{X}}+\alpha\left(\mathrm{u}^{\mathrm{ex}}, \mathrm{v}\right)_{\mathrm{X}}=(\mathrm{d}, \mathrm{Av})_{\mathrm{Y}} \forall \mathrm{v} \in \mathrm{X}$

Combining (36) and (37) we get :

$\left(\mathrm{A}\left(\mathrm{u}^{(\alpha)}-\mathrm{u}^{\mathrm{ex}}\right), \mathrm{Av}\right)_{\mathrm{Y}}+\alpha\left(\mathrm{u}^{(\alpha)}-\mathrm{u}^{\mathrm{ex}}, \mathrm{v}\right)_{\mathrm{X}}+\alpha\left(\mathrm{u}^{\mathrm{ex}}, \mathrm{v}\right)_{\mathrm{X}}=\left(\mathrm{d}-\mathrm{d}^{\mathrm{ex}}, \mathrm{Av}\right)_{\mathrm{Y}} \quad \forall \mathrm{v} \in \mathrm{X}$

Take $v=u^{(\alpha)}-u^{e x}$, one obtains

$\left|A\left(u^{(\alpha)}-u^{e x}\right)\right|^{2}+\alpha\left\|u^{(\alpha)}-u^{e x}\right\|^{2}=\left(d-d^{e x}, A\left(u^{(\alpha)}-u^{e x}\right)\right)_{Y}$

$-\alpha\left(u^{e x}, u^{(\alpha)}-u^{e x}\right)_{X}$

The last term can be written as

$\alpha\left(u^{e x}, u^{(\alpha)}-u^{e x}\right)_{X}=\alpha\left(A^{*} d^{1}, u^{(\alpha)}-u^{e x}\right)_{X}=\alpha\left(d^{1}, A\left(u^{(\alpha)}-u^{e x}\right)\right)_{Y}$

The left hand side of $(38)$ is bounded by

$\left|\mathrm{A}\left(\mathrm{u}^{(\alpha)}-\mathrm{u}^{\mathrm{ex}}\right)\right|^{2}+\alpha \| \mathrm{u}^{(\alpha)}-\mathrm{u}^{\mathrm{ex}}||^{2} \leq\left|\mathrm{d}-\mathrm{d}^{\mathrm{ex}}\right| \cdot\left|\mathrm{A}\left(\mathrm{u}^{(\alpha)}-\mathrm{u}^{\mathrm{ex}}\right)\right|+$ $\alpha\left|\mathrm{d}^{\mathrm{l}}\right| \cdot\left|\mathrm{A}\left(\mathrm{u}^{(\alpha)}-\mathrm{u}^{\mathrm{ex}}\right)\right| \leq\left|\mathrm{A}\left(\mathrm{u}^{(\alpha)}-\mathrm{u}^{\mathrm{ex}}\right)\right| \cdot\left(\left|\mathrm{d}-\mathrm{d}^{\mathrm{ex}}\right|+\alpha\left|\mathrm{d}^{\mathrm{l}}\right|\right)$

$\leq\left|\mathrm{A}\left(\mathrm{u}^{(\alpha)}-\mathrm{u}^{\mathrm{ex}}\right)\right|^{2}+(1 / 4)\left(\left|\mathrm{d}-\mathrm{d}^{\mathrm{ex}}\right|+\alpha\left|\mathrm{d}^{\mathrm{l}}\right|\right)^{2}$

Finally, by using $|\mathrm{d}-\mathrm{d} \operatorname{ex}|<\varepsilon$ and $\alpha=\mathrm{k} \varepsilon$, one obtains the inequality (35)

$\alpha\left\|u^{(\alpha)}-u^{e x}\right\|^{2} \leq(1 / 4)\left(\left|d-d^{e x}\right|+\alpha\left|d^{1}\right|\right)^{2}$

$\Rightarrow\left\|\mathrm{u}^{(\alpha)}-\mathrm{u}^{\mathrm{ex}}\right\| \leq(1 / 2) \alpha^{-1 / 2}\left(\left|\mathrm{~d}-\mathrm{d}^{\mathrm{ex}}\right|+\alpha\left|\mathrm{d}^{\mathrm{l}}\right|\right)$

\section{Geometry Bound Methods for Static Problems}

It is often of interest to determine, not exactly the geometry of defects and cracks, but only their approximate location in the solid. Geometry bound methods consist of determining spatial subdomains which do not contain internal defects and cracks. The initial inverse problem of determining defects or cracks in $\Omega$, using the boundary data pairs $\left\{u, \partial_{n} u\right\}$ for Laplace's 
equation, or $\{\mathbf{u}, \mathbf{T}[\mathbf{u}]\}$ for elasticity, is replaced by a set of inverse problems for smaller and smaller domains $\Omega$ ', with new data pairs to be determined for $\partial \Omega$ '. The condition to be checked is that the spatial domain $\mathrm{Z}$ between $\mathrm{S}$ (exterior boundary of $\partial \Omega$ ) and $\mathrm{S}$ ' (exterior boundary of $\partial \Omega^{\prime}$ ) must be free of defects.

Mathematically, for elliptic equations such as the Laplace equation, the transfer of the boundary data pair $\left\{u, \partial_{n} u\right\}$ from $S$ to $S$ ' is called analytical continuation or Cauchy's problem. It is not the purpose of the present paper to make an extensive review of Cauchy's problems for elliptic equations, in particular for the Laplace equation. There are some important mathematical works devoted to such problems, Laurentiev (1967), Lattès and Lions (1967). We shall mention only some of them, particularly a new approach suitable for applications in engineering problems, which makes use of explicit computations using transfer matrix operators for solving inverse problems. Different methods can be used for solving Cauchy Problems.

1. Trial and error of the boundary S'. For the trial S', solve the Cauchy problem in Z, then use of an analyticity criterion to check the continuation inside $Z$. In the example of distributed damage, $\mathbf{c}(\mathbf{x})$ must be zero inside $Z$. Equivalently, the defect indicator must be zero for the boundary $\partial Z=\left\{S,-S^{\prime}\right\}$. Two methods allow the determination of the solutions inside $Z$, the Quasi-reversibility Method introduced in (Lattès and Lions, 1967) and the Moment Method (Dang-Dinh et al, 2002).

2. Step-by-step continuation of the field from $\mathrm{S}$ to the inside until the appearance of singularities (cracks, discontinuity of gradients etc). The change from $S$ to $S$ ' is infinitesimal and the solution is obtained by means of the Transfer Matrix Operator (Bui, 1993, 1994). The second method is suitable for numerical solutions to static and transient problems.

\subsection{Ill-posedness of the Cauchy Problem}

Let us illustrate some methods for 2D geometries. Consider an inverse problem for a square with the superabundant data $\mathrm{u}=1$ and $\mathrm{g}:=\partial_{\mathrm{n}} \mathrm{u}=-1$ given on $\mathrm{x}_{2}=0$, while $\mathrm{u}=\mathrm{l}+\mathrm{x}_{2}$ on the lateral boundaries $x_{1}=0$ and $x_{1}=1$. Find $u$ and the normal derivative $\partial_{2} u$ on the side $x_{2}=1$. The analytic solution is $u\left(x_{1}, x_{2}\right)=1+x_{2}$. This classical example given by Hadamard (1952) illustrates the illposedness of the inverse problem.

Let us assume that the first boundary value $u=1$ on $x_{2}=0$ is perturbed by a small noise of the sin-form

$$
\mathrm{u}\left(\mathrm{x}_{1}, 0\right)=1+\varepsilon \sin \left(2 \mathrm{k} \pi \mathrm{x}_{1}\right)
$$

with small wave length $1 / \mathrm{k}$, and small amplitude $\varepsilon$. At a first sight, the additional term is small in the sense that both $1 / \mathrm{k}$ and $\varepsilon$ are small. But the small perturbation yields a large change of the expected normal gradient $\partial_{2} \mathrm{u}$ on $\mathrm{x}_{2}=1$ given by

$$
\partial_{2} \mathrm{u}\left(\mathrm{x}_{1}, 1\right)=1+2 \mathrm{k} \pi \varepsilon \sin \left(2 \mathrm{k} \pi \mathrm{x}_{1}\right) \sinh (2 \mathrm{k} \pi)
$$

This gradient blows up exponentially as $\mathrm{k} \rightarrow \infty$. This phenomenon illustrates the sensitivity of the solution to data errors. In the case of identification of a crack from Cauchy's data, 
without any constraint on the geometry of the crack, one can expect an oscillatory solution under small perturbation of data (star-like geometry, sea urchin geometry etc.). To limit the oscillatory solution on the defect geometry, one considers the constraints such as bounded length, smooth curve, etc to be imposed as a penalty on the error functional in optimisation methods. The determination of cracks by solving Cauchy's problems is considered in the paper (Alessandrini et al, 1999), where the best possible stability estimates are given. For the determination of an unknown smooth crack $\Gamma$ in $2 \mathrm{D}$, with Dirichlet condition, one assumes that the length of the crack is bounded. This is an a priori knowledge which is a constraint for regularizing the Cauchy problem. A stability result of the type $\mathrm{d}_{H}\left(\Gamma, \Gamma^{\prime}\right) \leq \mathrm{A}(\log |\log \varepsilon|)^{-1 / 2}$, with $\varepsilon$ the error in Cauchy's data, is given in (Alessandrini, 1993). The "distance » between two solutions $\Gamma, \Gamma^{\prime}$ is the Hausdorff distance between two curves

$$
\mathrm{d}_{\mathrm{H}}\left(\Gamma, \Gamma^{\prime}\right)=\max \left\{\sup _{\mathbf{x} \in \Gamma} \operatorname{dist}\left(\mathbf{x}, \Gamma^{\prime}\right), \sup _{\mathbf{x}^{\prime} \in \Gamma^{\prime}} \operatorname{dist}\left(\mathbf{x}^{\prime}, \Gamma\right)\right\}
$$

The regularisation procedures for solving the Cauchy problems are specific for each case. In next sections, we make a review of some methods for static inverse problems.

\subsection{The QR Method}

The Quasi-Reversibility method (Lattès and Lions, 1967) applies to a variety of problems, governed by elliptic, parabolic equations etc.

Consider the Cauchy problem for the Laplace equation. The QR method relies upon the use of a higher-order (4th order) partial differential equation defined herafter, depending on a small parameter $\varepsilon$, whose solution $\mathrm{u}(\varepsilon)$ is identical to the harmonic function $u$ in the domain $\Omega$, except in a layer of thickness $2 \varepsilon$.

Let us consider a square domain $\Omega:=\left\{\mathbf{x} \mid 0<x_{1}<1,0<x_{2}<1\right\}$. Consider $\Omega(2 \varepsilon):=\{\mathbf{x} \mid$ $\left.0<\mathrm{x}_{2}<1-2 \varepsilon\right\}, \mathrm{C}(\varepsilon):=\left\{\mathbf{x} \mid 1-\varepsilon<\mathrm{x}_{2}<1\right\}$. The domains $\Omega(2 \varepsilon)$ and $\Omega$ share a common boundary denoted by $\Gamma$, where the data are given $u=u^{d}$ and $g:=\partial_{n} u=g d$.

The Cauchy problem is equivalent to the problem of finding $u(\varepsilon)$ solution of the problem :

$$
\begin{aligned}
& \Delta\left(\mathrm{M}^{(\varepsilon) 2} \Delta \mathrm{u}^{(\varepsilon)}\right)=0 \\
& \mathrm{u}(\varepsilon)=\mathrm{u}^{\mathrm{d}} \text { and } \mathrm{g}(\varepsilon):=\partial_{\mathrm{n}} \mathrm{u}(\varepsilon)=\mathrm{gd} \text { on } \Gamma
\end{aligned}
$$

where $\mathrm{M}^{(\varepsilon)}(\mathbf{x})$ is a $\mathrm{C}^{2}$ continuous and positive function defined in $\Omega \square$ by :

$$
\begin{array}{ll}
M(\varepsilon)\left(\mathrm{x}_{1}, \mathrm{x}_{2}\right)=1 & \mathrm{x} \in \Omega(2 \varepsilon) \\
\mathrm{M}(\varepsilon)\left(\mathrm{x}_{1}, \mathrm{x}_{2}\right)=0 & \mathrm{x} \in \mathrm{C}(\varepsilon)
\end{array}
$$

and $M(\varepsilon)\left(x_{1}, x_{2}\right)=0$ continuous for $1-2 \varepsilon<x_{2}<1-\varepsilon$. Since $M(\varepsilon)\left(x_{1}, 1\right)=0$, no other conditions are required for $\mathrm{u}(\varepsilon)$ and $\mathrm{v}$ on the boundary $\mathrm{x}_{2}=1$. 
The uniqueness of the solution $u(\varepsilon)$ as well as the $\mathrm{L}^{2}$-convergence $u(\varepsilon) \rightarrow \mathrm{u}$ are proved in (Lattès and Lions, 1967). Finally, the ill-posed Cauchy Problem is replaced by a well-posed one using a higher-order equation.

No explicit regularization procedures are needed because the functions space $\mathrm{H}^{2}(\Omega)$ considered in the variational formulation of the fourth order equation (40) are implicitly more regular than the space $\mathrm{H}^{1}(\Omega)$ considered in the original variational of the second order equation. The variational problem consists of finding $\mathrm{u}(\varepsilon) \in \mathrm{X}, \mathrm{X}$ being the set of function in $\mathrm{H}^{2}(\Omega)$ satisfying (41) such that :

$$
\left.\left(\mathrm{M}^{(\varepsilon)} \Delta \mathrm{u}^{(\varepsilon)}\right), \mathrm{M}^{(\varepsilon)} \Delta \mathrm{v}\right)=0, \text { for any } \mathrm{v} \in \mathrm{X}^{(0)}
$$

and $\mathrm{X}^{(0)}$ being the set of test functions $\mathrm{v} \in \mathrm{H}^{2}(\Omega)$ satisfying $\mathrm{v}=0$ and $\partial_{\mathrm{n}} \mathrm{v}=0$ on $\Gamma$. A better result can be obtained by using the penalty form of the variational equation

$$
\left.\left(\mathrm{M}^{(\varepsilon)} \Delta \mathrm{u}^{(\varepsilon)}\right), \mathrm{M}^{(\varepsilon)} \Delta \mathrm{v}\right) / \varepsilon^{2}+\left(\mathrm{r}^{(\varepsilon)} \operatorname{gradu}(\varepsilon), \mathrm{r}^{(\varepsilon)} \operatorname{grad} \mathrm{v}\right)=0 \text { for any } \mathrm{v} \in \mathrm{X}(0)
$$

In the above equations the duality (.,.) means the integration over $\Omega(\varepsilon)$ of the product of dual terms. The function $\mathrm{r}^{(\varepsilon)}$ is defined by any continuous function such that $\mathrm{r}^{(\varepsilon)}=1$ for $\mathrm{x}_{2}<1-\varepsilon$, and $r^{(\varepsilon)}=\left(1-x_{2}\right) / \varepsilon$ for $1-\varepsilon<x_{2}<1$. The $L^{2}$-convergence $u^{(\varepsilon)} \rightarrow u$, to the solution $u$ of the Cauchy problem, is given in Lattès and Lions (1967).

\subsection{The Moment Method}

There is an important class of Cauchy problems for the Laplace equation with discrete superabundant data pairs given on the boundary. Consider a 2D Cauchy problem of determining the flux $\mathrm{g} \equiv \partial_{\mathrm{n}} \mathrm{u}$ on a known interior boundary $S_{\text {int }}$ from data partially known on the exterior boundary $S_{\text {ext }}$. The flux $g$ is known on the exterior boundary $S_{\text {ext }}$ and the potential $u$ is known on a bounded sequence of points $\left(\mathrm{x}_{1}, \mathrm{y}_{1}\right),\left(\mathrm{x}_{2}, \mathrm{y}_{2}\right), . .,\left(\mathrm{x}_{\mathrm{n}}, \mathrm{y}_{\mathrm{n}}\right)$ on a line segment $\mathrm{L}_{0}$ of the exterior boundary. The boundary $\partial \Omega$ consists of the exterior boundary $S_{\text {ext }}$ and the interior boundary $S_{\text {int }}$. Measurements performed in a sequence of points may be the best possible way for gathering experimental data.

This problem is solved by the moment method (Dang-Dinh Ang et al, 2002). The uniqueness of the solution of the inverse problem is proved in the latter reference which makes use of the boundary integral representation of harmonic functions with the Neumann type

$$
u(x, y)=a+\int_{S_{\text {ext }}} N(x, y ; p, q) g(p, q) d S(p, q)+\int_{S_{\text {int }}} N(x, y ; p, q) g(p, q) d S(p, q)
$$

where $a$ is a constant and $\mathrm{N}(\mathrm{x}, \mathrm{y} ; \mathrm{p}, \mathrm{q})$ the Neumann function for $\Omega$. Let $\mathrm{L}_{0}$ be defined by $\mathrm{y}=\mathrm{k}$. Substituting the values $\left(x_{n}, y_{n}=k\right), n=1,2,3 \ldots$ for the sequences of points in $L_{0}$ in $(41)$ and putting the unknowns a and $z(t)=g(\xi(t), \eta(t))$, with the parameter $t \in[0,1]$ describing the interior boundary $S_{\text {int }}$ in the 1.h.s. and known quantities $d$ in the r.h.s., one gets the observation equation settings in Hilbert spaces $\mathrm{X}, \mathrm{Y}$ with the linear map A : 


$$
\mathrm{A}(\mathrm{a}, \mathrm{z})=\mathrm{d}(\mathrm{a}, \mathrm{z}) \in \mathrm{X}, \quad \mathrm{d} \in \mathrm{Y}
$$

$-X$ is the Hilbert space of unknowns $u:=(a, z), X=\left\{(a, z): a \in R, z(t) \in H^{1}(0,1), z(0)=z(1)\right\}$, where $z(t)=g(p(t), q(t))$, and $(p(t), q(t)), t \in[0,1]$ describes the interior boundary $S_{\text {int }}$. The norm in $X$ is $\|(a, z)\|_{X}=\left(a^{2}+\|z\|^{2}\right)^{1 / 2}$.

$-\mathrm{Y}$ is the data space of sequence numbers

$$
\begin{gathered}
d:=\left\{d_{n}\right\}_{n \geq 1} \in Y \\
d_{n}=(1 / n)\left(u\left(x_{n}, k\right)-\int_{S_{e x t}} N\left(x_{n}, k ; p, q\right) g(p, q) d S(p, q)\right), \\
|d|=\left(\sum_{n \geq 1}\left|d_{n}\right| 2^{1 / 2<\infty}\right.
\end{gathered}
$$

$-\mathrm{A}(\mathrm{a}, \mathrm{z})$ is the sequence of numbers

$$
A(a, z):=\left\{(1 / n)\left(a+\int_{[0,1]} N\left(x_{n}, k ; p(t), q(t)\right)\left(\left|p^{\prime}(t)\right|^{2}+\left|q^{\prime}(t)\right|^{2} \mid\right)^{1 / 2} z(t) d t\right)\right\}_{n \geq 1} \in Y
$$

Equation (42) has been put in the standard form (27) or in the variational form (28) for which Tikhonov's regularisation procedure (29) applies. Detailed study of the Moment method can be found in the paper (Dang-Dinh Ang et al, 2002), where the error estimate of the regularized solution with the regularised parameter $\alpha$ proportional to the data error $\varepsilon$ is found to be $\mathrm{O}\left(\varepsilon^{1 / 2}\right)$.

\subsection{The Transfer Matrix Method}

Let us consider the same square domain $\Omega:=\left\{\mathbf{x} \mid 0<x_{1}<1,0<x_{2}<1\right\}$ for solving the Cauchy problem with superabundant data $\left(\mathrm{u}=\mathrm{u}^{\mathrm{d}}\right.$ and $\left.\mathrm{g}:=\partial_{\mathrm{n}} \mathrm{u}=\mathrm{g}^{\mathrm{d}}\right)$ on $\mathrm{x}_{2}=1,0<\mathrm{x}_{1}<1$ and the homogeneous Neumann condition $\partial_{\mathrm{n}} \mathrm{u}=0$ on $\mathrm{x}_{1}=0$ or $\mathrm{x}_{1}=1,0<\mathrm{x}_{2}<1$. The Laplace equation is written in the form :

$$
\begin{aligned}
& \partial_{2} u=g \\
& \partial_{2} g:=\partial_{2} \partial_{2} u=-\partial_{1} \partial_{1} u
\end{aligned}
$$

Let $z=(u, g)^{\tau}$ be the vector with components $u, g(\tau$ : transposition symbol). We can rewrite the Laplace equation in the differential form, with respect to $x_{2}$ :

$$
\begin{gathered}
\left(\partial / \partial \mathrm{x}_{2}\right) \mathrm{z}=\mathrm{Az} \\
\mathrm{A}_{11}=0, \quad \mathrm{~A}_{12}=1, \quad \mathrm{~A}_{21}=-\partial_{1} \partial_{1}, \quad \mathrm{~A}_{22}=0
\end{gathered}
$$

In the above, $A=\left(A_{i j}\right)$ is the "Transfer Matrix » operator acting on the vector $z=(u, g) \tau$ defined on the front $\Gamma\left(x_{2}=\right.$ constant $)$.

Let us interpret (43) as the rate of change of the data pair $(u, g)$ when the front $\Gamma\left(x_{2}=t\right)$ moves upwards as $t$ increases. The time-like parameter $t$ describes the position of $\Gamma$, moving in 
the $\mathrm{Ox}_{2}$ direction with the unit velocity $\mathrm{V}=1$. Therefore we can have an Eulerian interpretation of (43) as the particle velocity of $z$ or " material » derivative $d z / d t=V \partial z / \partial x_{2}$ of the function $z$ which does not depend explicitly on $\mathrm{t}, \partial \mathrm{z} / \partial \mathrm{t}=0$, on the moving $\Gamma_{\mathrm{t}}$

$$
\mathrm{dz} / \mathrm{dt}=\mathrm{Az}
$$

Naively, one can attempt to integrate the Cauchy system (45) by the " time » integration with « initial» conditions $\mathrm{z}_{0}=\left(\mathrm{u}^{\mathrm{d}}, \mathrm{g}^{\mathrm{d}}\right)^{\tau}$ on $\Gamma_{\mathrm{t}=0}$. One difficulty relies on the presence of an differential operator with respect to the complementary variable $x_{1}$ along $\Gamma$ and associated boundary conditions on $\partial \Gamma$. Another difficulty relies on the ill-posedness of the Cauchy problem which is recovered in the differential system equation (45). We shall see later how the ill-posedness of the differential equation (45) can be overcame by specific regularisation procedures.

This new interpretation of the rate change of $\mathrm{z}$ makes it possible to generalize the transfer matrix to arbitrary shape front $\Gamma_{t}$, curved front in $2 \mathrm{D}$ or curved surface front in $3 \mathrm{D}$. It is convenient to consider that the front $\Gamma_{\mathrm{t}+\mathrm{dt}}$ is derived from the precedent one $\Gamma_{\mathrm{t}}$ by displacing each point in $\Gamma_{\mathrm{t}}$ by the quantity $\psi(\mathrm{x})$ ndt, where $\mathrm{n}$ is the unit normal vector to the front, $\psi(\mathrm{x})$ is a positive and regular scalar field representing the normal "velocity». Therefore the " material » derivative of $\mathrm{u}$ is given by $\mathrm{du} / \mathrm{dt}=\psi \mathrm{g}$. We have to complete the latter equation by calculating explicitly the "material » derivative of $\mathrm{dg} / \mathrm{dt}$, in the form $\mathrm{dg} / \mathrm{dt}=\mathrm{A}(\mathrm{u}, \mathrm{g}, \psi) \cdot(\mathrm{u}, \mathrm{g})^{\tau}$, with the matrix operator A. Let us introduce the following notations for tangential operators

$$
\begin{aligned}
& \operatorname{grad} \Gamma(.):=\operatorname{grad}(.)-\mathbf{n} \partial_{\mathrm{n}}(.) \quad(\text { tangential gradient }) \\
& \operatorname{div} \Gamma(.):=\operatorname{div}(.)-\mathbf{n} . \partial_{\mathrm{n}}(.) \quad(\text { tangential divergence })
\end{aligned}
$$

Now we remark that the function $u$ at any fixed point left behind the moving integration front $\Gamma_{\mathrm{t}}$ does not change its value $\partial \mathrm{u} / \partial \mathrm{t}=0$. This invariance condition expresses the continuation of function $\mathrm{u}$, which does not depend on $\mathrm{t}$ or $\Gamma_{\mathrm{t}}$.

Let us consider the physical domain $Z_{t}$ free of defects, between the initial front $\Gamma_{t=0}$, the current front $\Gamma_{t}$ and eventually the lateral surface $S_{L}$. We assume that $u$ satisfies the following equations (Problem P) :

$$
\begin{aligned}
& -\operatorname{div} \operatorname{grad} u=0 \quad \text { in } \Omega_{t} \\
& \partial_{\mathrm{n}} \mathrm{u}=\mathrm{g} \quad \text { on } \Gamma_{\mathrm{t}} \quad \text { (outward normal) } \\
& \partial_{\mathrm{n}} \mathrm{u}=\mathrm{gd} \quad \text { on } \Gamma_{0} \quad \text { (inward normal) } \\
& \partial_{n} u=0 \quad \text { on } S_{L}
\end{aligned}
$$

Three cases can be considered :

1. The lateral boundary $S_{L}$ does not exist, or $\partial \Gamma_{t}$ is void for any $t$,

2. The lateral boundary is orthogonal to $\Gamma_{\mathrm{t}}$ and $\partial_{\mathrm{n}} \mathrm{u}=0$ on $\mathrm{S}_{\mathrm{L}}$ as indicated in (49), 
3. The lateral boundary reduces to two fixed points ( $2 \mathrm{D}$ problem) or a close curve (3D problem)

The problem $(P)$ is equivalent to the classical variational problem $a_{t}(u, v)=b_{t}(v)$ for any admissible field $\mathrm{v}$

$$
\int_{\Omega_{t}} \operatorname{grad} u . g r a d v d \Omega=\int_{\Gamma_{t}} g v d S-\int_{\Gamma_{0}} g^{d} v d S
$$

where the test functions $\mathrm{v}$ are assumed to be independent of $\mathrm{t}$. Consider the Problem $\mathrm{P}$ ' obtained by differentiating (50) with respect to time

$$
(\mathrm{d} / \mathrm{dt}) \mathrm{a}_{\mathrm{t}}(\mathrm{u}, \mathrm{v})=(\mathrm{d} / \mathrm{dt}) \mathrm{b}_{\mathrm{t}}(\mathrm{v}) \quad \forall \mathrm{v},
$$

Here the time dependence of the bilinear form $a_{t}(u, v)$ and the linear form $b_{t}(v)$ comes solely from the variable integration domains, rather the functions themselves. Taking into account the invariance condition $\partial \mathrm{u} / \partial \mathrm{t}=0$ and Reynolds's formulae for convected differentiations of integrals and performing the integrations by parts of integrals, we arrive at the following results (Bui and Bonnet 1989, Bui 1993)

$$
\begin{gathered}
(\mathrm{d} / \mathrm{dt}) \mathrm{u}=\psi \mathrm{g} \\
(\mathrm{d} / \mathrm{dt}) \mathrm{g}=-\operatorname{div} \Gamma(\psi \operatorname{grad} \Gamma \mathrm{u})-\operatorname{gdiv} \Gamma(\psi \mathrm{n})
\end{gathered}
$$

The transfer matrix operator $\mathrm{A}$ is given by its components

$$
\mathrm{A}_{11}=0, \quad \mathrm{~A}_{12}=\psi, \quad \mathrm{A}_{21}=-\operatorname{div} \Gamma(\psi \operatorname{grad} \Gamma), \quad \mathrm{A}_{22}=-\operatorname{div} \Gamma(\psi \mathrm{n})
$$

The above method of derivation of the transfer matrix A for any elliptical equations can be applied to elasticity and to time harmonic acoustics (Bui, 1993). Since the operator A is unbounded, there is no inequality of the form $\|A z\| \leq C\|z\|$ with some $C>0$. Such an inequality is necessary for the existence and uniqueness of the solution of the differential equation.

Therefore the Cauchy system $\mathrm{dz} / \mathrm{dt}=\mathrm{Az}$ is unstable without a regularisation procedure which specifies the spaces of smooth functions used.

\subsection{Regularization methods}

Let us consider the Cauchy problem for the Laplace equation $-\operatorname{div}(\operatorname{gradu})=0$ on a square domain $0<x_{1}<1,0<x_{2}<1$ with the data pair $\left(u^{d=}=0, g^{d}=-x_{1}^{2}\right)$ on the side $x_{2}=0$ and the Neumann boundary $g=0$ on the lateral sides $x_{1}=0$ and $x_{1}=1$. We want to determine the flux $g$ on the upper side $\mathrm{x}_{2}=1$.

It is well known that, the numerical integration of (45) is unstable, even for small integration steps along the $\mathrm{Ox}_{2}$ axis. Numerically, the instability arises from poor calculation of the tangential second derivative which introduces errors of the Hadamard type which blows up with time and becomes worse when noise is present. Numerical result for the step size $\delta=0.02$ with the noise $\varepsilon=0.01$ shows instability after the first step integration, Figure (3a). 
A regularization procedure is required for obtaining stable solutions. Mathematically, for given functions $\mathrm{x}_{1} \rightarrow \mathrm{u}\left(., \mathrm{x}_{1}\right)$ one defines the smooth functions $\mathrm{x}_{1} \rightarrow \underline{u}\left(., \mathrm{x}_{1}\right) \equiv \mathrm{Mu}$ by using a smoothing operator $\mathbf{M}$, which can be defined in different equivalent manners (Lorentz and Andrieux, 2003). Since A involves second derivatives with respect to the spatial variable along $\Gamma_{\mathrm{t}}$ (the spatial variable is $\mathrm{x}_{1}$ in this example), we consider the space of smooth functions $\underline{\mathrm{u}}$ $\in \mathrm{H}^{2}([0,1]), \underline{u}^{\prime}\left(\mathrm{x}_{1}=0\right)=\underline{u}^{\prime}\left(\mathrm{x}_{1}=1\right)=0$, such that the operator $\mathrm{AM}$ is bounded, $\|A M z\| \leq \mathrm{C}^{\prime}\|\mathrm{z}\|$ for some constant $C^{\prime}$ and for $\mathrm{z}=\left(\underline{\mathrm{u}}, \partial_{2} \underline{u}\right)$. The following presentation of smoothing techniques for functions of $x_{1} \in[0,1]$ is similar to that given in the article (Lorentz and Andrieux, 2003):

\section{Differential equation:}

$\mathrm{M}: \mathrm{u} \rightarrow \mathrm{Mu}:=\underline{\mathrm{u}}$ solution of

$$
\begin{aligned}
& \underline{\mathrm{u}}-\mathrm{k}^{2} \underline{\mathrm{u}}^{\prime \prime}+\mathrm{h}^{4} \underline{\mathrm{u}}^{\prime, \prime}=\mathrm{u} \quad \text { in }[0,1] \\
& \underline{\mathrm{u}}(0)=\underline{\mathrm{u}} \cdot(1)=0, \underline{\mathrm{u}}^{\prime \prime}(0)=\underline{\mathrm{u}},{ }^{\prime},(1)=0,
\end{aligned}
$$

where $\mathrm{k}$ and $\mathrm{h}$ is a characteristic length, small with respect to the interval $[0,1]$ but large with respect to the wave length of the numerical oscillations.

Variational equation:

For given $\mathrm{u}$, find $\underline{\mathrm{u}} \in \mathrm{H}^{2}([0,1])$ such that

$$
\int_{[0,1]}\left[(\underline{u}-u) v+k^{2} \underline{u}^{\prime} v^{\prime}+h^{4} \underline{u}^{\prime}, v^{\prime} ’\right] d s=0, \quad \forall v \in H^{2}([0,1]),
$$

where $\mathrm{v}$ satisfies the boundary conditions (56). Test functions $\mathrm{v}$ satisfying (56) can be for example Fourier cosinus-functions of the form $v\left(x_{1}, x_{2}\right)=\sum_{n>1} a_{n}\left(x_{2}\right) \cos \left(n \pi x_{1}\right)$.

Minimisation of functional:

$$
\mathrm{u} \rightarrow \underline{\mathrm{u}}=\operatorname{Arg} \operatorname{Min}_{\mathrm{v} \in \mathrm{H}^{2}} \int_{[0,1]}\left[(\mathrm{v}-\mathrm{u})^{2}+\mathrm{k}^{2}\left(\mathrm{v}^{\prime}\right)^{2}+\mathrm{h}^{4}\left(\mathrm{v}^{\prime \prime}\right)^{2}\right] \mathrm{ds}
$$

The first term alone which corresponds to the least square approximation gives yet a good result because of the smoothness of the space used $\mathrm{H}^{2}$. However, better results are obtained with the fisrt additional term and which corresponds to the gradients penalty limiting higher order oscillations.

$$
\begin{aligned}
& \text { Convolution integral } \\
& \qquad \underline{\mathrm{u}} \equiv \mathrm{Mu}(\mathrm{x})=\int_{[0,1]}^{\mathrm{G}(\mathrm{x}, \mathrm{y}) \mathrm{u}(\mathrm{y}) \mathrm{dy},}
\end{aligned}
$$

$\mathrm{G}(\mathrm{x} ; \mathrm{y})$ : Green function solution of the equation $\mathrm{G}-\mathrm{k}^{2} \mathrm{G}^{\prime \prime}+\mathrm{h}^{4} \mathrm{G}^{\prime,},{ }^{\prime}=\delta(x-y)$. Finally, the Cauchy problem consists of the integration of the differential equation with bounded transfer operator AM, instead of the unbounded A :

$$
(d / d t) z=A M z
$$


and taking into account the initial condition $\mathrm{z}(\mathrm{t}=0)=\left(\mathrm{u}^{\mathrm{d}}, \partial_{\mathrm{n}} \mathrm{u}=\mathrm{g}^{\mathrm{d}}\right)^{\tau}$. At time $\mathrm{t}+\mathrm{dt}$, updated values of the vector $z$ on $\Gamma_{t+d t}$, are given by $\left.z(t+d t)=z(t)+A M z(t) d t\right)$. Since $A M$ is bounded, we have the inequality, $\|\mathrm{AMz}\| \leq \mathrm{C}\|\mathrm{z}\|$ for $\mathrm{z}=\left(\underline{\mathrm{u}}, \partial_{2} \underline{\mathrm{u}}\right)$ which guarantees the existence and uniqueness of the solution of the differential equation if the integration step is small enough.

\subsection{An example}

Let us consider an example of the inverse problem of the Laplace equation $\operatorname{div}(\mathrm{grad}) \mathrm{u}=0$, Figure (2). Find the normal derivative $g\left(x_{1}, 1\right)$ on the side $A D$, with given data pair $(u=0$, $\mathrm{g}=\mathrm{x}_{1}{ }^{2}$ ) on $\mathrm{BC}$ and with Dirichlet's boundary conditions given on $\mathrm{AB}$ and $\mathrm{CD}$ as indicated in Figure (2) (i.e. the homogeneous Neumann boundary condition).

We observe that with a noise $1 \%$ on the data pair along BC, the numerical solution at $x_{2}=2 \delta$ is spurious Figure (3a). The numerical result shows a high oscillation around its means value represented by the exact solution. A regularisation procedure is needed for eliminating oscillations in the space domain, by introducing low-pass filtering (55) or (58). For example, the result of an explicit integration of the regularised equation (59), using the penalty method (58) with $k \neq 0, h=0$, is shown in Figure (3b). Even with noised data of $2 \%$, the analytic solution is recovered within $2 \%$. In this example, all calculations are performed explicitly.

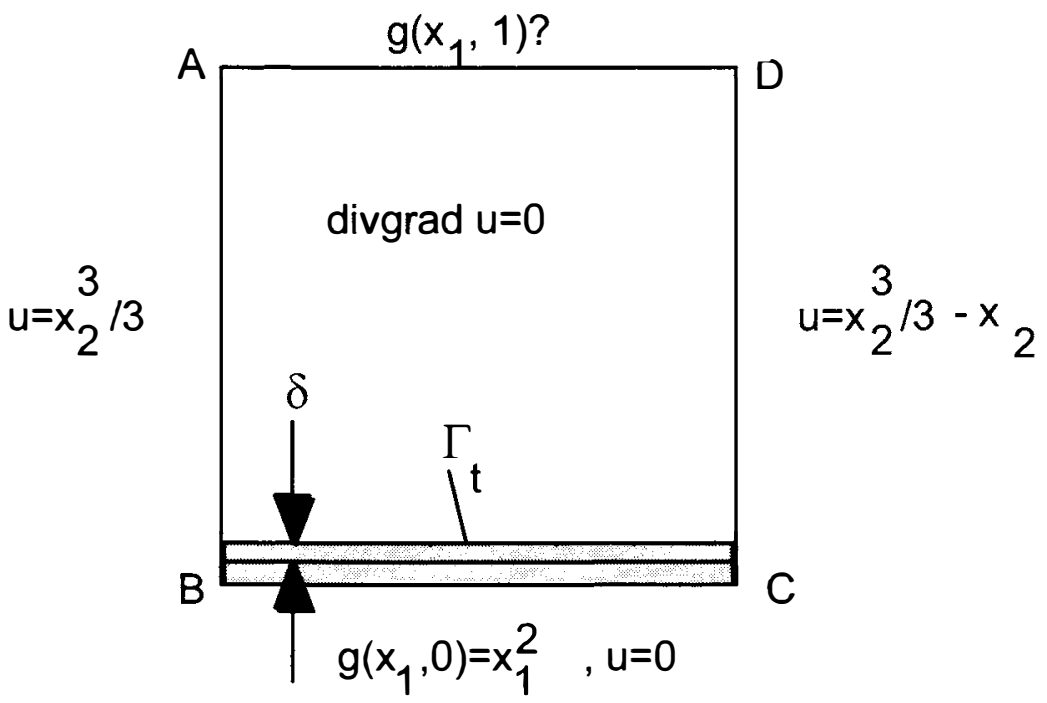

Figure 2. Integration of the Cauchy problem along the moving front $\Gamma_{\mathrm{t}}$ with step $\delta=0.02$. 

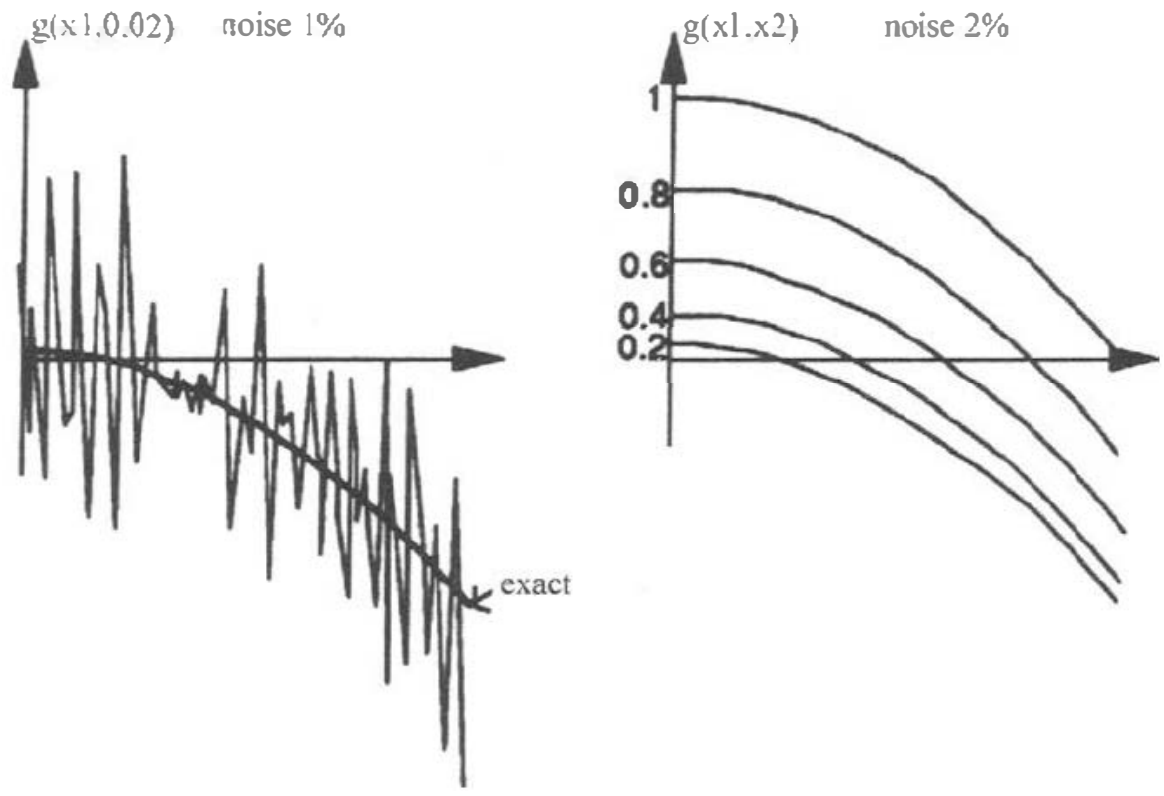

Figure 3. (a), (b). Numerical integration of the regularised system (60) (after Bui, 1993).

\section{Geometry Bound Methods for Dynamic Elasticity}

One natural way to detect internal defects consists of considering plane P-waves with different directions of propagation p. Suppose that the transducers are located at the same distance of the center of the solid and that $t=0$ records the initial time when the waves are emitted. If $t=t(\mathbf{p}, x)$ records the time when a plane wave $p$ arrives at some point $x$, the wave reflected by the defect $C$ at point $M$ and at time $T=t(p, M)$ is back to the transducer at time $2 T$, which can be recorded. The position of waves at times $T$ defines the exterior convex hull bounding the defect $C$.

Ultrasonic testing methods of evaluating of materials make use of the same principle. However, one has to assume that no perturbation of plane incident waves arises from the boundedness of the solid. Such method is impossible to be achieved experimentally because incident plane waves on a bounded body can be possible only if the load on $\partial \Omega$ can chosen appropriately (for example by imposing at any time the load corresponding to the restriction to $\partial \Omega$ of plane elastic wave travelling in infinite medium). Experimental applied loads on the boundary are not plane waves inside the solid. Earthquake signals can be considered as plane waves or spherical waves only when they are far fields. For such general waves, the method on construction of the convex hull $\mathrm{C}$ is based on the use of instantaneous Reciprocity Gap Functional as an indicator of defect, which detects directly the arrival time $T$.

The instantaneous Reciprocity Gap Functional in elastodynamics is defined as : 


$$
\mathrm{R}(\mathrm{t}):=\mathrm{R}(\mathrm{v}(\mathrm{t}))=\int_{S}\{\mathbf{n} \cdot \sigma[\mathbf{v}] \cdot \mathbf{u}-\mathbf{n} . \sigma[\mathbf{u}] . \mathbf{v}\} \mathrm{dS} \equiv \int_{\Sigma} \mathbf{n} \cdot \sigma[\mathbf{v}] \cdot[[\mathrm{u}]] \mathrm{d} S
$$

where $\mathbf{u}(\mathbf{x}, \mathbf{t})$ is the current elastodynamic field of the cracked body for a given initial and boundary data and $v(x, t)$ is the "adjoint fields" of the uncracked infinite body whose particular form is defined hereafter. Consider a plane shear wave (with Y(.) the Heaviside function)

$$
\mathbf{v}(\mathbf{x}, \mathrm{t})=\mathbf{k Y}(\mathbf{t}-\mathbf{x} \cdot \mathbf{p} / \mathrm{c}-\tau)
$$

propagating in the direction $\mathbf{p}$ with the shear wave velocity $\mathbf{c}$, with $\tau$ a parameter chosen in such a way that all waves $\mathbf{p}$ are outside the solid at $t=0$.

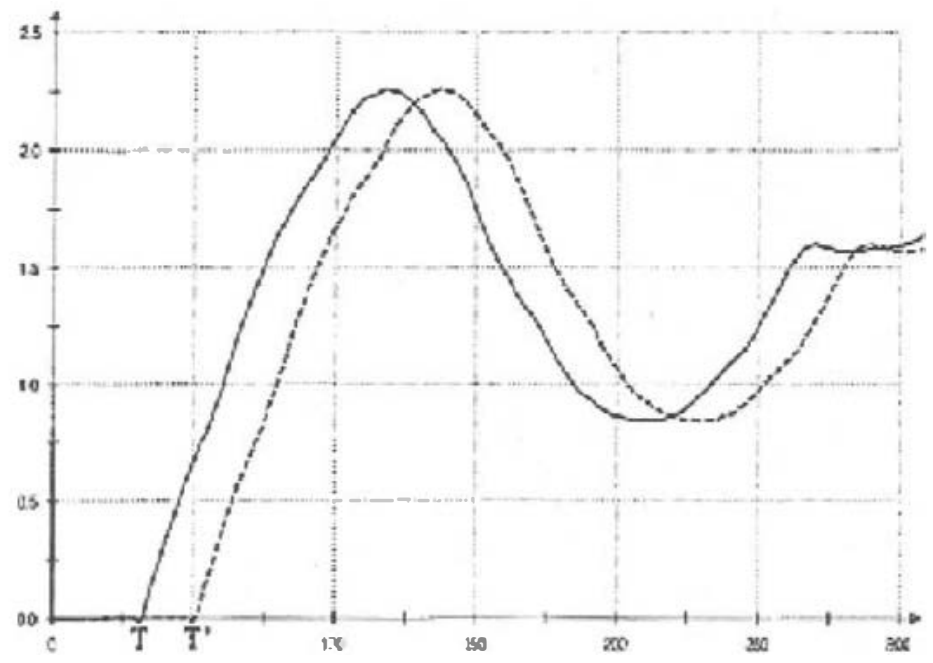

Figure 4. Instantaneous reciprocity gap $\mathbf{R}(t ; \mathbf{p})$ associated to adjoint wave $\mathbf{p}$. $T$ is the arrival time when the wave reaches the defect. $T$ ' is the arrival time of opposite adjoint wave -p. 


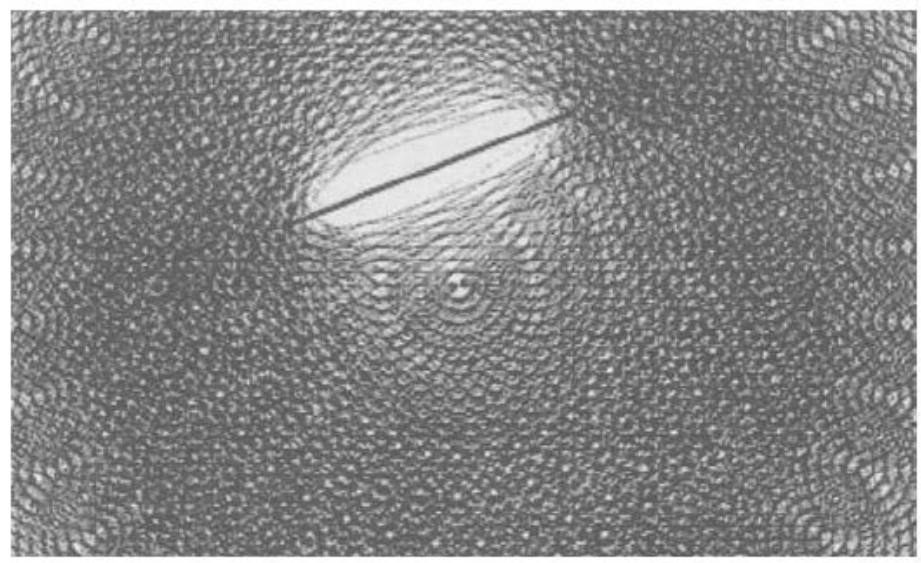

Figure 5. The convex hull containing the crack.

The stress $\sigma[\mathrm{v}]$ is a travelling Dirac delta impulse which does not produce any virtual work with the crack displacement $[[u]]$ until the adjoint field $v(x, t)$ interacts with the crack $\Sigma$.

Therefore the indicator $\mathrm{R}(\mathrm{t} ; \mathrm{p})$ vanishes identically for $\mathrm{t}<\mathrm{T}$ and begins to take a non zero value $\varepsilon$ after the time $T(p)$. For adjoint waves of opposite direction $-\mathbf{p}$, the starting time is $T$ ' as indicated in Figure (4). If we plot the wave fronts at different times until they reach the crack, because of the numerical error $\varepsilon$ in the detection of the contact point, we obtain the oval shaped hull $\mathrm{C}$ containing the crack, Figure 5.

The current elastodynamic field $\mathbf{u}(\mathbf{x}, \mathbf{t})$ considered in Figure 4 is obtained numerically for a sudden constant shear stress applied on the crack surface. The amount of the shear stress is opposite to the shear stress released in earthquake. The small errors on the evaluation of the time $T(p)$ at the crack tips have the order of one finite element and are justified by the fact that the crack tips do not open too much during the start of loading. Therefore the convex hull appears to be a flat oval. The load considered for the current elastodynamic field $\mathbf{u}(\mathbf{x}, \mathbf{t})$ simulates the earthquake signal due a sudden release of some (constant) shear stress acting on the fault. To determine the position and geometry of the fault and also the magnitude of the earth quake, defined by [[u]], we have to soive an inverse problem. The position and geometry of the fault can determined only by using direct computation of an indicator $R(t)$, with data available on the ground. By a double integration in time of the acceleration $\partial_{\mathbf{t}} \partial_{\mathbf{l}} \mathbf{u}$ we have the datum $\mathbf{u}(\mathbf{x}, \mathrm{t})$ on the boundary. The second knowledge of the data pair is given by the stress free condition $\sigma[\mathbf{u}] \cdot n=0$ on the ground and by the vanishing normal discontinuity $\left[\left[u_{n}\right]\right]=$ on the fault. Plane waves considered in $R(t)$ are for adjoint fields only.

\section{Exact Solutions for Planar Crack Identification}

As a matter of fact, the reciprocity gap functional (61) depending on the current field $\mathbf{u}$ and the adjoint field $v$ is a bilinear form on $\mathbf{u}, \mathbf{v}$ 


$$
\mathrm{R}(\mathbf{u} ; \mathbf{v})=\int_{S}\{\mathbf{n} \cdot \sigma[\mathbf{v}] \cdot \mathbf{u}-\mathbf{n} \cdot \sigma[\mathbf{u}] \cdot \mathbf{v}\} \mathrm{dS}
$$

Its value depends on the data pair $(\mathbf{u}, \mathbf{n} . \sigma[\mathbf{u}])$. By choosing appropriately the adjoint field, one can determine the unknown crack parameters, for example the discontinuity $[[\mathbf{u}]]$ and its support plane.

There is an analogy with the problem of determining an unknown $\mathbf{x}$ in the vector space $\mathrm{X}$ of dimension $\mathrm{N}$, knowing the bilinear forms $\mathrm{R}\left(\mathbf{x} ; \mathbf{e}^{\mathrm{i}}\right):=\left(\mathbf{x}, \mathbf{e}^{\mathrm{i}}\right), \mathrm{i}=1,2, . . \mathrm{N}$ which are nothing but the coordinates of the vector $\mathbf{x}$ in the basis of adjoint vectors $\left(\mathbf{e}^{\mathrm{i}}\right)$. The bilinear form $\mathrm{R}(\mathbf{u} ; \mathbf{v})$ provides a duality between the vector space $U$ of the current field $u$ (displacement or temperature etc) and the dual space $U^{*}$ of adjoint fields $v$ which satisfies the adjoint equations. The inverse problem for identifying the crack consists of finding the normal $\mathbf{n}$ to the crack plane, the position of the plane $\Pi$ defined by $x_{3}=c$ with $\mathrm{Ox}_{3}$ along the $\mathbf{n}$-direction, and the crack geometry $\Sigma$.

Difficulties in solving this class of inverse problems arise from the nonlinear dependence of $\mathbf{u}$ on the set of elements $\{\mathbf{n}, \mathbf{c}, \Sigma\}$, which belong to a metric space (where only the distance between elements can be defined). The reciprocity gap functional provides a new method for identifying explicitly the unknowns $\{\mathbf{n}, \mathbf{c}, \Sigma\}$. It consists of choosing appropriately the loading conditions (e.g the fields $\mathbf{u}$ ) and the adjoint fields $\mathbf{v}$ in such a way that the boundary data pair provide explicit valued functions $\mathrm{R} d(\mathrm{v}) \equiv \mathrm{f}(\mathbf{n}, \mathrm{c}, \Sigma)$ of the unknowns $\{\mathbf{n}, \mathrm{c}, \Sigma\}$, instead of functionals of $\mathbf{u}$ (which depends implicitly on these unknowns). Since adjoint functions satisfy the equations for uncracked body, it is a simpler task to find appropriate fields which can reveal the unknowns.

Hereafter, we give applications for elliptic, parabolic and hyperbolic equations.

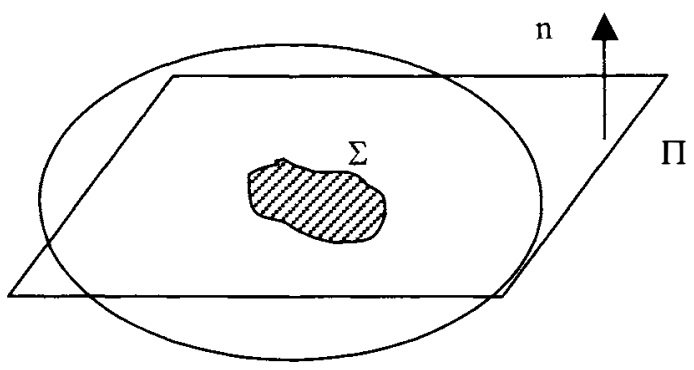

Figure 6. Planar crack in solid.

\subsection{Planar crack in 3D elasticity}

Andrieux and Ben Abda (1992) were among the first to determine explicitly cracks in 2D inverse problems using the Reciprocity Gap Functional, which is widely used in the literature for another mathematical context (uniqueness or identifiability), for the Laplace equation, 
Friedmann and Vogelius (1989), Alessandrini (1988), Bryan and Vogelius (1992). Their method of solution was extended to 3D quasi-static elasticity for determining the host plane of the planar crack, Andrieux, Ben Abda (1996). The complete solution for quasi-static elasticity including the crack shape was given in Andrieux, Ben Abda and Bui (1997), (1999).

The current field equations for the displacement field $\mathbf{u}(\mathbf{x})$ is :

$$
\begin{array}{cc}
\operatorname{div}(\sigma[\mathbf{u}])=\mathbf{0} & \text { in } \Omega \backslash \Sigma \\
\sigma[\mathbf{u}]=\mathrm{A} \cdot \operatorname{grad}(\mathbf{u}) & \text { in } \Omega \backslash \Sigma \\
\sigma[\mathbf{u}] . \mathbf{n}=\mathbf{F}^{\mathrm{d}} \quad \text { on } \mathrm{S} \text { (exterior boundary) } \\
\sigma[\mathbf{u}] \cdot \mathbf{n}=\mathbf{0} & \text { on } \Sigma \quad \text { (crack surfaces) } \\
\mathbf{u}=\mathbf{u}^{\mathrm{d}} & \text { on } \mathrm{S} .
\end{array}
$$

The data pair ( $\mathbf{u}^{\mathrm{d}}, \mathbf{F}^{\mathrm{d}}$ ) are assumed to be known and compatible and the vector $\mathbf{F}^{\mathbf{d}}$ is assumed to satisfy the self-equilibrium relationships. The Reciprocity Gap Functional provides the equation

$$
\int_{\Sigma} \mathbf{n} \cdot \sigma[\mathbf{v}] \cdot[[\mathbf{u}]] \mathrm{dS}=\int_{S}\left\{\mathbf{n} \cdot \sigma[\mathbf{v}] \cdot \mathbf{u}^{\mathrm{d}}-\mathbf{F} \mathrm{d} \cdot \mathbf{v}\right\} \mathrm{dS}
$$

(A different sign is found in Andrieux, Ben Abda and Bui (1997), (1999), Ben Abda and Bui (2003) because opposite normal is used here to define the jump; $\mathbf{n}$ denotes here the normal to the lower crack surface). Denoting by the same symbol [[u]] the extension of the jump to the whole host plane $\Pi$, by letting it equal to zero outside $\Pi$, we can re-write (63) as

$$
\int_{\Pi} \mathbf{n} \cdot \sigma[\mathbf{v}] \cdot[[\mathbf{u}]] \mathrm{d} S=\int_{S}\left\{\mathbf{n} \cdot \sigma[\mathbf{v}] \cdot \mathbf{u}^{\mathrm{d}}-\mathbf{F} \mathrm{d} \cdot \mathbf{v}\right\} \mathrm{dS}
$$

We denote the left hand side of $(64)$ by $R(\mathbf{u}, \mathbf{v})$ and the right hand side by $\mathrm{R}^{\mathrm{d}}(\mathbf{v})$. Here the adjoint field $v$ satisfes the same elasticity equation in the whole space domain without crack. We assume that the elastic moduli tensor $\mathrm{A}$ is isotropic, with $\mathrm{E}$ and $v$ being the Young modulus and the Poisson ratio respectively. One can interpret the linear map $\mathbf{v} \rightarrow R(\mathbf{u}, \mathbf{v})$ as a linear continuous form on the space $\mathrm{L}^{2}(\Pi)$ which determines completely [[u]] (Riesz's representation theorem). We show now that particular adjoint fields determine explicitly the host plane and the jump support.

\section{Determination of the normal}

We introduce the following (ij)-family of adjoint displacement field $\mathbf{v}^{(\mathrm{ij})}$, the k-component of which is defined by : 


$$
\left.\mathrm{v}^{(\mathrm{ij}}\right)_{\mathrm{k}}=\left(\mathrm{A}^{-1}\right)_{\mathrm{kl}} \mathrm{mnEij}_{\mathrm{mn}} \mathrm{Xl}_{\mathrm{l}}
$$

with $E^{i j} j_{m n}=\left(\delta i_{m} \delta i_{n}+\delta{ }_{n} \delta i_{m}\right) / 2$ and $A^{-1}$ the compliance tensor (inverse of $\left.A\right)$. The indices take values in the set $\{1,2,3\}$ and $\delta j_{n}$ is the Krönecker delta. The adjoint stress field $\sigma[\mathrm{v}(\mathrm{ij})]=A \varepsilon\left[\mathrm{v}^{(\mathrm{ij})}\right]=\mathrm{E}(\mathrm{ij})$ with components $\mathrm{Eij}_{\mathrm{mn}}$ is constant.

Denoting by $\mathrm{Q}_{\mathrm{ij}}=\mathrm{R}(\mathbf{u}, \mathbf{v}(\mathrm{ij}))=\mathrm{R} \mathrm{d}(\mathbf{v}(\mathrm{ij}))$ the second order symmetric tensor, equal also to

$$
\mathrm{Q}=\left(\mathbf{n} \otimes \int_{\Pi}[[\mathbf{u}]] \mathrm{dS}\right)_{(\mathrm{sym})}
$$

the following results hold:

$$
\begin{gathered}
\left|\int_{\Pi}[[\mathbf{u}]] \mathrm{dS}\right|=\left\{2 \mathrm{Q}_{\mathrm{ij}} \mathrm{Q}_{\mathrm{ij}}-\mathrm{Q}_{\mathrm{hh}} \mathrm{Q}_{\mathrm{kk}}\right\}^{1 / 2} \\
\int_{\Pi}\left[\left[\mathrm{u}_{\mathrm{n}}\right]\right] \mathrm{dS}=\mathrm{Q}_{\mathrm{ii}} \\
\left|\int_{\Pi}\left[\left[\mathbf{u}^{(\mathrm{t})}\right]\right] \mathrm{dS}\right|=\left\{2 \mathrm{Q}_{\mathrm{ij}} \mathrm{Q}_{\mathrm{ij}}-2 \mathrm{Q}_{\mathrm{hh}} \mathrm{Q}_{\mathrm{kk}}\right\}^{1 / 2}
\end{gathered}
$$

where $\left[\left[u_{n}\right]\right]=[[\mathbf{u}]] . n$ is the normal jump (a scalar) and $\left[\left[\mathbf{u}^{(t)}\right]\right]$ is the tangential jump vector $\left[\left[\mathbf{u}^{(t)}\right]\right]=[[\mathbf{u}]]-([[\mathbf{u}]] . \mathbf{n}) \mathbf{n}$. The tensor $\mathrm{Q}_{\mathrm{ij}}$ is known by its data value $\mathrm{Q}_{\mathrm{ij}}=\mathrm{R}^{\mathrm{d}}\left(\mathbf{v}^{(\mathrm{ij})}\right)$, given by the right hand side of (63) for the boundary data of the current field and the adjoint field $\mathbf{v}^{(\mathrm{ij})}(65)$.

We assume that the applied loadings are such that the mean value of the total jump does not vanish,

$$
\left\{2 \mathrm{Q}_{\mathrm{ij}} \mathrm{Q}_{\mathrm{ji}}-\mathrm{Q}_{\mathrm{hh}} \mathrm{Q}_{\mathrm{kk}}\right\}^{1 / 2} \neq 0
$$

The last assumption can be checked with the data pair and the boundary conditions of the adjoint field, which can be changed if necessary to satisfy the required conditions. Later, we shall show how to practically satisfy the latter assumption.

As a result, the unit vectors $\mathbf{n}$ and $\mathbf{U}=\int_{\Pi}[[\mathbf{u}]] \mathrm{dS} /\left|\int_{\Pi}[[\mathbf{u}]] \mathrm{dS}\right|$ can be determined by considering the normalized tensor Q'

$$
Q_{i j}^{\prime}=R^{d}\left(v^{(i j)}\right)\left\{2 Q_{i j} Q_{j i}-Q_{h h} Q_{k k}\right\}^{-1 / 2}
$$

the two non-vanishing principal values of which are $\lambda_{1}=\left(1+Q^{\prime}{ }_{h h}\right) / 2$ and $\lambda_{2}=\left(1-Q^{\prime}{ }_{h h}\right) / 2$. All possible vectors $\mathbf{n}$ and $\mathbf{U}$ can be chosen by the permutation and the change of signs in the following pairs of vectors, in the basis of eigenvectors $\left(\Phi^{1}, \Phi^{2}, \Phi^{3}\right)$ of the tensor Q'

$$
\left\{.\left[\sqrt{ } \lambda_{1}, \sqrt{ }-\lambda_{2}, 0 .\right]\left[\lambda_{1},-\sqrt{ }-\lambda_{2}, 0\right]\right\}
$$


It is necessary that two different loadings (or current fields) (a) and (b) are used for defining the tensors $\mathrm{Q}^{(\mathrm{a})}$ and $\mathrm{Q}^{(\mathrm{b})}$ in order to determine the normal as the product of their third eigenvectors

$$
\mathbf{n}=\left(\Phi^{3}(\mathrm{a}) \times \Phi^{3(b)}\right)\left|\Phi^{3}(\mathrm{a}) \times \Phi^{3(b)}\right|^{-1}
$$

We recover the result already obtained by Alessandrini and Diaz Valenzuala (1994) about the number $t w o$ of loadings necessary and sufficient for crack identification.

\section{Determination of the crack plane}

Once the normal $\mathbf{n}$ has been identified, let $\mathrm{Ox}_{3}$ be chosen in the $n$-direction, $(\mathbf{T}, \mathbf{V}, \mathbf{n})$ be the orthonormal direct basis vectors. We can identified the crack plane by $\mathrm{x}_{3}+\mathrm{C}=0$ and determine the constant $C$ by choosing adjoint fields $v^{\alpha}, \alpha=T, V$, such that the stress field $\left.n . \sigma\left[\mathbf{v}^{\alpha}\right)\right]$ is linear in $\mathrm{x}_{3}$. The equation (63) or (64) provides thus a linear equation in the form $\mathrm{pC}+\mathrm{q}=0$, where the constants $\mathrm{p}$ and $\mathrm{q}$ are known. The following adjoint fields $v^{\alpha}, \alpha=T, V$ have shear components $\sigma_{31}$ and $\sigma_{32}$ linear in $\mathrm{x}_{3}$.

$$
\begin{aligned}
& v_{1}(T)=-x_{1}^{2 / 2 E}-v x_{2}^{2 / 2 E}+(2+v) x_{3}^{2 / 2 E}, \quad v_{2} T=v x_{1} x_{2} / E, \quad v_{3} T=v x_{1} x_{3} / E \\
& v_{1}(v)=-x_{2}^{2 / 2 E}-v x_{1} 2 / 2 E+(2+v) x_{3} 2 / 2 E, \quad v_{2} V=v x_{1} x_{2} / E, \quad v_{3} V=v x_{2} x_{3} / E
\end{aligned}
$$

These fields activate the tangential component $\int_{\Pi}\left[\left[\mathbf{u}^{t}\right]\right] \mathrm{dS}$, so that we need a second assumption on the actual field $\left\{2 \mathrm{Q}_{\mathrm{ij}} \mathrm{Q}_{\mathrm{ji}}-2 \mathrm{Q}_{\mathrm{hh}} \mathrm{Q}_{\mathrm{kk}}\right\}^{1 / 2} \neq 0$ for determining $\mathrm{C}$, given by

$$
|C|=\left\{R^{(T) 2}+R^{(V) 2}\right\}^{1 / 2}\left\{2 Q_{i j} Q_{j i}-2 Q_{h h} Q_{k k}\right\}^{-1 / 2}
$$

where $\mathrm{R}(\mathrm{T}):=\mathrm{R}^{\mathrm{d}}(\mathbf{v}(\mathrm{T})), \mathrm{R}(\mathrm{V}):=\mathrm{R}^{\mathrm{d}}(\mathbf{v}(\mathrm{V}))$. Since the crack normal $\mathbf{n}$ is known, the second assumption $\left\{2 \mathrm{Q}_{\mathrm{ij}} \mathrm{Q}_{\mathrm{ji}}-2 \mathrm{Q}_{\mathrm{hh}} \mathrm{Q}_{\mathrm{kk}}\right\} 1 / 2 \neq 0$ can be easily satisfied by taking the following loading $\mathbf{F d}^{\mathrm{d}}=\mathbf{n} . \boldsymbol{\sigma}\left[\mathbf{v}^{(\mathrm{S})}\right]$ for the adjoint field as well for the cracked body, where $\mathbf{v}^{(\mathrm{S})}$ is a pure shear displacement field with the non zero stress components $\sigma[\mathbf{v}(\mathrm{S})]_{\mathrm{tn}}=\sigma[\mathbf{v}(\mathrm{S})]_{\mathrm{nt}}=$ constant. The tangential jump $[[\mathbf{u}]]^{(t)} \neq 0$ does not vanish because the conditions $\mathbf{n} \cdot \sigma[\mathbf{u}] \cdot \mathbf{n}=0$ on the crack and $[[\mathbf{u}]](t)=0$, already satisfied by the adjoint field $\mathbf{v}(\mathrm{S})$, should correspond to a current field identical to $\mathbf{v}(\mathrm{S}), \mathbf{u} \equiv \mathbf{v}(\mathrm{S})$. This contradicts the assumption that the reciprocity gap $\mathrm{R}^{\mathrm{d}}\left(\mathbf{v}^{(\mathrm{S})}\right)$ does not vanish. Therefore, for pure shear loading, we see that the second assumption is true $\left\{2 \mathrm{Q}_{\mathrm{ij}} \mathrm{Q}_{\mathrm{ji}}-2 \mathrm{Q}_{\mathrm{hh}} \mathrm{Q}_{\mathrm{kk}}\right\}^{1 / 2} \neq 0$, so the first assumption must be, $\left\{2 \mathrm{Q}_{\mathrm{ij}} \mathrm{Q}_{\mathrm{ji}}-\mathrm{Q}_{\mathrm{hh}} \mathrm{Q}_{\mathrm{kk}}\right\}^{1 / 2} \neq 0$, because $\left\{2 \mathrm{Q}_{\mathrm{ij}} \mathrm{Q}_{\mathrm{ji}}-\mathrm{Q}_{\mathrm{hh}} \mathrm{Q}_{\mathrm{kk}}\right\}^{1 / 2}>\left\{2 \mathrm{Q}_{\mathrm{ij}} \mathrm{Q}_{\mathrm{ji}}-2 \mathrm{Q}_{\mathrm{hh}} \mathrm{Q}_{\mathrm{kk}}\right\}^{1 / 2}>0$.

\section{Determination of the crack geometry}

Crack shape determination in 3D inverse elastic problem is given the first time in Andrieux, Ben Abda and Bui $(1997,1999)$. The complete identification of the crack geometry is based on the use of adjoint fields which generalized to elasticity the well-known adjoint fields introduced by Calderon (1980) for inverse steady state conduction problem. 
Let us remark that the support supp $[[\mathbf{u}]]$ in the $\Pi$ plane coincides with the crack surface $\Sigma$. A rigorous proof of this theorem can be found in Andrieux, Ben Abda and Bui $(1997,1999)$ and is not reproduced here.

The key method for the identification of the jump [[u]] consists of evaluating its 2D Fourier transform in the $\Pi$ plane. Take the origin $O$ and the base vectors $\mathbf{e}^{1}, \mathbf{e}^{2}$ on the plane $\Pi$, and $\mathbf{n} \equiv \mathbf{e}^{3}$ normal to the plane $\Pi$, we introduce a $2 \mathrm{D}$ parameter $\mathbf{k}=\left(\mathrm{k}_{1}, \mathrm{k}_{2}, 0\right)$ and two complex $3 \mathrm{D}$ fields of vectors in $\mathrm{R}^{3}+i \mathrm{R}^{3}$, depending on $\mathbf{k}$ and $\mathbf{n}=(0,0,1)$

$$
\begin{array}{r}
\mathbf{Z}_{(\mathbf{k})}=\left(\mathbf{k}+\mathrm{i}|\mathbf{k}| \mathbf{e}^{3}\right) \\
\mathbf{Z}_{(\mathrm{k})}^{*}=\left(\mathbf{k}-\mathrm{i}|\mathbf{k}| \mathbf{e}^{3}\right)
\end{array}
$$

Generalizing Calderon (1980), we introduce two k-families of adjoint fields

$$
\begin{aligned}
& \mathbf{w}^{+}(\mathbf{x} ; \mathbf{k})=\nabla_{\mathrm{x}} \exp \left(-\mathrm{i} \mathbf{Z}_{(\mathrm{k})} \cdot \mathbf{x}\right)+\nabla_{\mathrm{x}} \exp \left(-\mathrm{i} \mathbf{Z}_{(\mathrm{k})}^{*} \cdot \mathbf{x}\right) \\
& \mathbf{w}^{-}(\mathbf{x} ; \mathbf{k})=\nabla_{\mathrm{x}} \exp \left(-\mathrm{i} \mathbf{Z}_{(\mathrm{k})} \cdot \mathbf{x}\right)-\nabla_{\mathrm{x}} \exp \left(-\mathrm{i} \mathbf{Z}_{(\mathbf{k})}^{*} \cdot \mathbf{x}\right)
\end{aligned}
$$

In (75) and (76) the gradient in the $\left(\mathrm{x}_{1}, \mathrm{x}_{2}, \mathrm{x}_{3}\right)$-space is denoted by $\nabla_{\mathrm{x}}$. The adjoint stress fields associated to (75) and (76) are in equilibrium $\operatorname{div}\left(\sigma\left[\mathbf{w}^{ \pm}\right]\right)=0$ and infinitely differentiable everywhere in $\Omega$.

The reciprocity gaps take the forms

$$
\begin{gathered}
\mathrm{R}\left(\mathbf{u}, \mathbf{w}^{+}(\mathbf{k})\right)=(2 \mathrm{E} /(1+v))|\mathbf{k}|^{2}\left[\left[\mathrm{u}_{3}\right]\right]^{\mathrm{F}}(\mathbf{k}) \\
\mathrm{R}\left(\mathbf{u}, \mathbf{w}^{-}(\mathbf{k})\right)=-(2 \mathrm{E} /(1+v)) \mathrm{i}|\mathbf{k}| \mathbf{k} \cdot\left[\left[\mathbf{u}^{(\mathrm{t})}\right]\right]^{\mathrm{F}}(\mathbf{k})
\end{gathered}
$$

where $\left[\left[u_{3}\right]\right]^{F}(\mathbf{k})$ is the Fourier transform of the normal component $\left[\left[u_{3}(\mathbf{x})\right]\right]$ (a complex scalar) and $\left[\left[\mathbf{u}^{(t)}\right]\right]^{F}(\mathbf{k})$ the Fourier transform of the tangential jump $\left[\left[\mathbf{u}^{(t)}(\mathbf{x})\right]\right]$ (a 2D complex vector). From the boundary value $\mathrm{R}^{\mathrm{d}}\left(\mathbf{w}^{+}(\mathbf{k})\right)$ of the reciprocity gap with $\mathbf{w}^{+}$, we get explicitly the Fourier transform of the normal component $\left[\left[\mathrm{u}_{3}\left(\mathrm{x}_{1}, \mathrm{x}_{2}\right)\right]\right]$

$$
\left[\left[\mathrm{u}_{3}\right]\right]^{\mathrm{F}}(\mathbf{k})=((1+v) / 2 \mathrm{E})|\mathbf{k}|^{-2} \mathrm{R}^{\mathrm{d}}\left(\mathbf{w}^{+}(\mathbf{k})\right)
$$

As a matter of fact, $\mathbf{w}^{+}(\mathbf{k})$ and $\mathrm{R}^{\mathrm{d}}\left(\mathbf{w}^{+}(\mathbf{k})\right)$ which is linear in $\mathbf{w}^{+}(\mathbf{k})$, behave like $\mathrm{O}(|\mathbf{k}|)$ as $|\mathbf{k}| \rightarrow 0$ when considered as functions of $\mathbf{k}$. Therefore the r.h.s of (79) has a simple pole at $\mathbf{k}=\mathbf{0}$ in the $\left(\mathrm{k}_{1}, \mathrm{k}_{2}, 0\right)$-plane, and thus admits a 2D-Fourier inverse tranform. With a suitable choice of the boundary data, such that $\mathrm{R}^{\mathrm{d}}\left(\mathbf{w}^{+}(\mathbf{k})\right) \neq 0$, equation (79) provides a non-zero crack opening displacement $\left[\left[\mathrm{u}_{3}\left(\mathrm{x}_{1}, \mathrm{x}_{2}\right)\right]\right] \neq 0$ and solves the inverse problem. The quantity $\mathrm{R}^{\mathrm{d}}\left(\mathbf{w}^{+}(\mathbf{k})\right)$ is a crack 
opening indicator. A non vanishing value $\mathrm{R}^{\mathrm{d}}\left(\mathbf{w}^{+}(\mathbf{k})\right) \neq 0$ can be checked explicitly, using its definition $R^{d}(v)$ in (62). An altemative solution is provided by (78), with the boundary value of the reciprocity gap $\mathrm{R}^{\mathrm{d}}\left(\mathbf{w}^{-}(\mathbf{k})\right)$. We can write (78) as

$$
+i k_{1}\left[\left[u_{1}\right]\right]^{\mathrm{F}}(\mathbf{k})+i k_{2}\left[\left[u_{2}\right]\right]^{\mathrm{F}}(\mathbf{k})=-((1+v) / 2 \mathrm{E})|\mathbf{k}|^{-1} \mathrm{R}^{\mathrm{d}}\left(\mathbf{w}^{-}(\mathbf{k})\right)
$$

The r.h.s of (80) is known from the data, while the 1.h.s of (80) is nothing but the Fourier transform of the divergence of the tangential jump vector $\operatorname{div}\left(\left[\left[\mathbf{u}^{(t)}\right]\right]\right)$, the support of which is also the crack surface.

Consequently, whenever the tangential displacement indicator $\mathrm{R}^{\mathrm{d}}\left(\mathbf{w}^{-}(\mathbf{k})\right) \neq 0$ does not vanish (near $\mathbf{k}=\mathbf{0}$ it behaves like $\mathrm{O}\left(|\mathbf{k}|^{2}\right.$ ), equation (80) solves the crack shape identification problem by a 2D-Fourier inverse transform of a known function of $\mathbf{k}$.

\subsection{Planar crack identification for the transient heat equation}

This problem arises in non-destructive thermal testing of materials using infra-red measurements. Up to our knowledge, theoretical results on identifiability of cracks are scarce in the transient case. They are generally restricted to a steady-state case corresponding to the Laplace equation, which is a simpler problem already solved for 3D planar crack by Andrieux and Ben Abda (1992). The solution for transient case has been solved by Ben Abda and Bui (1999) (Some misprints are present in the latter reference). Let us consider the equation for the current thermal field $\mathrm{u}(\mathbf{x}, \mathrm{t})$, with normalized material constant

$$
\begin{array}{lr}
\partial_{\mathrm{t}} \mathrm{u}-\Delta \mathrm{u}=0 & \text { in } \Omega \backslash \Sigma \times[0, \mathrm{~T}] \\
\mathrm{u}(\mathbf{x}, \mathrm{t})=0, & \mathrm{t} \leq 0 \\
\mathrm{u}=\mathrm{u}^{\mathrm{d}} & \text { on } \mathrm{S} \times[0, \mathrm{~T}] \\
\partial_{\mathrm{n}} \mathrm{u}=0 & \text { on the crack } \Sigma
\end{array}
$$

The direct problem (81)-(84) of determining the field $u(x, t)$ is a well-posed one. The inverse problem consists of determining the crack $\Sigma$ using the datum $u^{d}$ together with the measurement of the temperature flux $\Phi^{\mathrm{d}}$

$$
\partial_{\mathrm{n}} \mathrm{u}(\mathbf{x}, \mathbf{t})=\Phi^{\mathrm{d}} \quad \text { on } \mathrm{S} \times[0, \mathrm{~T}]
$$

The reciprocity gap functional $\mathrm{R}(\mathrm{u}, \mathrm{w})$ is the bilinear form

$$
R(u, w)=\int_{[0, T]} \int_{S}\left(u^{d} \partial_{n} w-\Phi^{d} w\right) d S d t
$$


where the field w satisfies the adjoint equations

$$
\begin{gathered}
-\partial_{\mathrm{t}} \mathrm{w}-\Delta \mathrm{w}=0 \quad \text { in } \Omega \times[0, \infty] \\
\mathrm{w}(\mathbf{x}, \mathrm{t} \rightarrow \infty)
\end{gathered}
$$

The reciprocity gap is related to the temperature jump $[[u]]$ on the crack by

$$
\int_{[0, \infty]} \int_{\Sigma}[[u]] \partial_{n} w d S d t=\int_{[0, \infty]} \int_{S}\left(u d \partial_{n} w-\Phi^{d} w\right) d S d t
$$

Additional conditions for the validity of (89) are conceming the behavior at large time of $\mathrm{u}^{\mathrm{d}} \partial_{\mathrm{n}} \mathrm{w}$ and $\Phi^{\mathrm{d}} \mathrm{w}$ which must vanish as $\mathrm{t} \rightarrow \infty$. To satisfy these additional conditions one can consider adjoint functions $w$ which vanish for $\mathrm{t}>\mathrm{T}$. A better choice consists of considering " diffusive» boundary conditions for the current field $u$, rather than imposing (88) to the adjoint field.

Diffusive boundary conditions. If after some time $t_{1}$ we consider the thermal boundary condition such that $u^{d}\left(x, t>t_{1}\right) \leq K \exp (-\lambda t) v(x), x \in S$, where $v$ is the (positive) eigenfunction associated to the eigenvalue $\lambda$ of the harmonic equation $-\Delta v=\lambda v$ with the homogeneous conditions $v=0$ on the exterior boundary and $\partial_{n} v=0$ on the crack. Therefore

$$
\begin{gathered}
\left(\partial_{\mathrm{t}}-\Delta\right)\{\mathrm{u}-\mathrm{Kexp}(-\lambda \mathrm{t}) \mathrm{v}(\mathbf{x})\}=0, \quad \mathbf{x} \in \Omega, \mathrm{t}>\mathrm{t}_{1} \\
\left\{\partial_{\mathrm{n}} \mathrm{u}-\mathrm{K} \exp (-\lambda \mathrm{t}) \mathrm{v}(\mathbf{x})\right\}=0, \quad \mathbf{x} \text { on the crack, } \mathrm{t}>\mathrm{t}_{1} \\
\{\mathrm{u}-\mathrm{K} \exp (-\lambda \mathrm{t}) \mathrm{v}(\mathbf{x})\} \leq 0, \quad \mathrm{x} \text { on the exterior boundary and } \mathrm{t}>\mathrm{t}_{1}
\end{gathered}
$$

After the parabolic minimum principle applied to the function $\{\mathrm{u}-\operatorname{Kexp}(-\lambda \mathrm{t}) \mathrm{v}(\mathrm{x})\}$ we have $\{\mathrm{u}-\operatorname{Kexp}(-\lambda \mathrm{t}) \mathrm{v}(\mathbf{x})\} \leq 0$, for $\mathbf{x} \in \Omega, \mathrm{t}>\mathrm{t}_{1}$. Hence the current temperature field is a function of exponential decay satisfying the required « diffusive » boundary condition.

Solution of the inverse problem. The method of solution of the inverse problem is similar to that is given in Section 7.1. That is to determine subsequently first the normal $\mathbf{n}$ to the crack plane, then the position of the plane and finally the crack shape, defined as the support of $[[\mathrm{u}]]$ in the crack plane by using suitable adjoint fields. Consider an adjoint function depending on a $3 \mathrm{D}$ vector $\mathbf{p}$ in the form $w(\mathbf{x} \cdot \mathbf{p}, t)$ defined hereafter. Let $\mathrm{R}^{\mathrm{d}}(\mathbf{p})=\mathrm{R}(\mathrm{w}(\mathbf{x} \cdot \mathbf{p}, \mathrm{t}))$ be the reciprocity gap given by the r.h.s of (89). Equation (89) can be written as

$$
\int_{[0, T]} \int_{\Sigma}[[u]] \partial_{n} w d S d t \equiv\left(n_{i} p_{i}\right) \int_{[0, T]} \int_{\Sigma}[[u]] w^{\prime}(\mathbf{x} \cdot \mathbf{p}, t) d S d t=R d(p)
$$


where $w^{\prime}(\mathbf{x} . \mathbf{p}, t)$ is the partial derivative $\partial_{y} w(\mathbf{x} \cdot \mathbf{p}, t)$ with respect to the first argument $\mathbf{y}=\mathbf{x} . \mathbf{p}$. We get an equation of the form

$$
\left(\mathbf{n}_{\mathrm{i}} \mathbf{p}_{\mathrm{i}}\right) \mathrm{A}(\mathbf{p})=\mathrm{R} \mathrm{d}(\mathbf{p})
$$

Another vector $\mathbf{q}$, linearly independent of $\mathbf{p}$, provides an additionnal equation

$$
\left(n_{i} q_{i}\right) A(\mathbf{q})=R d(\mathbf{q})
$$

which, together with (91) and $|\mathbf{n}|=1$, for given $\mathbf{p}$ and $\mathbf{q}$, determines the unknown unit normal $\mathbf{n}$ (Remark that $A(\mathbf{p})$ and $A(\mathbf{q})$ does not vanish since the reciprocity gap is not identically equal to zero for any $w$. Assume here that $\mathbf{p}$ and $\mathbf{q}$ can be chosen so that the unknowns $A(\mathbf{p}), A(\mathbf{q})$ can be normalized).

Another method for determining the normal is given in an Exercice. Also, the best way to determine $\mathbf{n}$ is to consider $\mathbf{p}$ and $\mathbf{q}$ such that $\mathrm{R}^{\mathrm{d}}(\mathbf{p})=\mathrm{R}^{\mathrm{d}}(\mathbf{q})=0$, by studying the zeros of $\mathrm{R}^{\mathrm{d}}(\mathbf{p})$. Such adjoint fields does not "see" the crack. Therefore $\mathbf{n}=\mathbf{p} \times \mathbf{q}$.

In (91), (92) an adjoint function $w$ for $\mathrm{R}^{\mathrm{d}}(\mathbf{p}), \mathrm{R}^{\mathrm{d}}(\mathbf{q})$, satisfying (87) and (88) can be the function

$$
\begin{array}{cr}
w(p)(x, t)=(4 \pi(T-t))^{-1 / 2} \exp \left\{-\left(x_{i} p_{i}\right)^{2 / 4}(T-t)\right\} & t<T \\
w(p)(x, t)=0 & t \geq T .
\end{array}
$$

For diffusive field $\mathrm{u}$, a simpler adjoint function can be simply $w(\mathrm{p})(\mathbf{x}, \mathrm{t})=(\mathrm{l} / 2)\left(\mathrm{x}_{\mathrm{i}} \mathrm{p}_{\mathrm{i}}\right)^{2}-|\mathbf{p}|^{2} \mathrm{t}$. Once the normal to the host plane is determined, let us operate a frame change with $\mathrm{Ox}_{3}$ along $n$ so that the crack plane is $x_{3}-C=0$. To determine the constant $C$, we choose the b-family of adjoint field $w^{(b)}(\mathbf{x}, t)$ independent of $x_{1}, x_{2}$

$$
\mathrm{w}^{(\mathrm{b})}(\mathbf{x}, \mathrm{t})=(4 \pi(\mathrm{T}-\mathrm{t}))^{-1 / 2} \exp \left\{-\left(\mathrm{x}_{3}-\mathrm{b}\right)^{2 / 4}(\mathrm{~T}-\mathrm{t})\right\} \quad \mathrm{t}<\mathrm{T}
$$

The corresponding reciprocity gap is written as

$$
\mathrm{R}(\mathrm{w}(\mathrm{b})(\mathbf{x}, \mathrm{t}))=(\mathrm{l} / 2)(\mathrm{b}-\mathrm{C}) \int_{[0, \mathrm{~T}]} \int_{\Sigma}[[\mathrm{u}]](4 \pi)^{1 / 2}(\mathrm{~T}-\mathrm{t})^{-3 / 2} \exp \left\{-(\mathrm{C}-\mathrm{b})^{2} / 4(\mathrm{~T}-\mathrm{t})\right\} \mathrm{dSdt}
$$

Hence the simple zero at $b=C$ of the function $b \rightarrow R d(b) \equiv R(w(b)(x, t))$, given by the r.h.s of (95) with the adjoint field (94), solves the inverse problem. Practically, one draws the curve $\mathrm{b} \rightarrow \mathrm{R}^{\mathrm{d}}(\mathrm{b})$ and determines the constant $\mathrm{C}$ as the simple zero of the curve.

We now determine the crack shape by studying the jump $D(x, t) \equiv[[u]]$. Instead of $(83)$ we consider the data on $\mathrm{S} \times[0, \infty]$, and we assume a rapid decay of $\mathrm{u}^{\mathrm{d}}$ at $\mathrm{t} \rightarrow \infty$. We denote by [[u]] the extension of the temperature jump to the whole plane $\Pi$ by letting $[[u]]=0$ outside the crack surface. We choose an adjoint function, depending on a $2 \mathrm{D}$ parameter $\mathbf{s}=\left(\mathrm{s}_{1}, \mathrm{~s}_{2}, 0\right)$ and a real positive scalar $\mathrm{q}>0$

$$
\mathrm{w}(\mathrm{s}, \mathrm{q})(\mathrm{x}, \mathrm{t})=\exp (\mathrm{iqt}) \exp (-\mathrm{is.x}) \exp \left(\mathrm{x}_{3}\left(\mathrm{~s}_{1}{ }^{2}+\mathrm{s}_{2}{ }^{2}-\mathrm{iq}\right)^{1 / 2}\right)
$$


Let $\mathrm{H}(\mathbf{x} ; \mathrm{q})=\int_{[0, \infty]}[[\mathrm{u}]] \exp (\mathrm{iqt}) \mathrm{dt}$. As functions of $\mathbf{x}=\left(\mathrm{x}_{1}, \mathrm{x}_{2}, 0\right), \mathrm{H}(\mathbf{x} ; \mathrm{q})$ and $[[\mathrm{u}]](\mathbf{x} ; \mathrm{t})$ have the same spatial support. Therefore we proceed to determine the support of the function $\mathbf{x}$ $\rightarrow \mathrm{H}(\mathbf{x} ; \mathrm{q})$. From the reciprocity gap relationship, we get $(\mathrm{q}>0)$

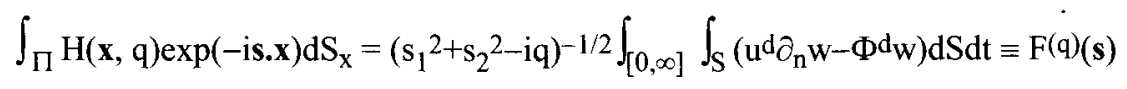

For fixed $\mathrm{q}>0$, the r.h.s. of (97) denoted simply by $\mathrm{F}(\mathrm{q})(\mathbf{s})$ is a smooth function of $\mathbf{s}$. Denote by $F(z)$ the complex extension of $F(q)(s)$ to the complex planes $z=\left(z_{1}, z_{2}\right)=\left(s_{1}+i v_{1}, s_{2}+i v_{2}\right)$. The singularities of $\mathrm{F}(\mathrm{z})$ do not meet the real axes. Observing that the solid $\Omega$ is bounded (embedded in a sphere $|\mathbf{x}| \leq a$ ) we obtain the majoration for large $|z|=\left(\left|z_{1}\right| 2+\left|z_{2}\right| 2\right)^{1 / 2}$

$$
|\mathrm{F}(\mathrm{z})| \leq \mathrm{C}|\mathrm{z}|^{-1} \exp \left(\mathrm{a}\left(\left|\mathrm{z}_{1}\right|+\left|\mathrm{z}_{2}\right|\right)\right) \exp \left(\mathrm{a}\left(\left|\mathrm{z}_{1}\right| 2+\left|\mathrm{z}_{2}\right| 2\right)^{1 / 2}\right) \leq \mathrm{Cexp}\left(2 \mathrm{a}\left(\left|\mathrm{z}_{1}\right| 2+\left|\mathrm{z}_{2}\right| 2\right)^{1 / 2}\right)
$$

where $\mathrm{C}$ is a constant depending on the time Fourier transforms of $\mathrm{u}^{\mathrm{d}}(\mathrm{t})$ and $\Phi^{\mathrm{d}}(\mathrm{t})$, i.e on $\mathrm{q}$.

Now, (98) expresses that $\mathrm{F}(\mathrm{z})$ is a function of $2 \mathrm{D}$ complex variables $\mathrm{z}$ of the exponential type 2a. A function $\mathrm{F}(\mathrm{z})$ is of the «exponential type $\mathrm{k} »$ if $|\mathrm{F}(\mathrm{z})| \leq \mathrm{Cexp}(\mathrm{k}|\mathrm{z}|)$, (L. Schwartz, 1978). Therefore $F(z)$ belongs to the space of tempered distribution $S^{\prime}\left(s_{1}, s_{2}\right)$. After the PaleyWiener theorem, the function $\mathrm{F}\left(\mathrm{s}_{1}, \mathrm{~s}_{2}\right)$ is the $2 \mathrm{D}$ spatial Fourier transform of a compact support function $\mathrm{H}(\mathbf{x}, \mathrm{q})$, the support of which is the crack surface.

Exercice. Take $\mathbf{p}=\mathbf{n} \times \mathbf{m}$, with unit orthogonal vectors $\mathbf{n}, \mathbf{m}$, show that the normal $\mathbf{n}$ is the solution of the MinMax problem (Andrieux 1995).

Proof: Let $\mathbf{p}$ and $\mathbf{q}=\mathbf{p}+\mathrm{d} \mathbf{p}$ be chosen so that $\mathrm{dR}=0$ for any increment $\mathrm{d} \mathbf{p}$. Now, the condition $\mathrm{dR}=0$, for any $\mathrm{d} \mathbf{n}$ and $\mathrm{d} \mathbf{m}$ subjected to the constraints $\mathbf{n} \cdot \mathbf{m}=0$, $|\mathbf{n}|=|\mathbf{m}|=1$ turns out to be the stationary condition for $\mathrm{R}(\mathbf{n} \times \mathbf{m})$, giving $\mathbf{n}$ as the solution of the MinMax problem

$$
\mathbf{n}=\arg \operatorname{Min}_{\mid \mathbf{n}} \mid=1\left\{\operatorname{Max}_{\mid \mathbf{m}} \mid=1, \mathbf{n . m}=0 \quad \mathrm{R}(\mathbf{n} \times \mathbf{m})\right\} \bullet
$$

\subsection{Solution of the Inverse scattering of planar crack in 3D transient acoustics}

Most studies of inverse acoustic scattering for identifying a crack assumed very special conditions : infinite medium, incident plane wave on the solid, analysis in the frequency domain.

We shall consider the general input loading by arbitrary boundary conditions applied to a bounded solid and analyse the equations in the time domain. The explicit solution derived in Bui, Constantinescu and Maigre (1999) is based on the reciprocity gap functional method.

We consider the acoustic wave equation with normalized constants and with (for a pure mathematical reason) a small damping term which will be set to zero in the limit process $\varepsilon>0$, $\varepsilon \rightarrow 0$

$$
\mathrm{L}[\mathrm{u}] \equiv\left(\partial_{\mathrm{t}} \partial_{\mathbf{t}}-\Delta+\varepsilon \partial_{\mathrm{t}}\right) \mathrm{u}=0 \quad \text { in } \Omega \times[0, \infty[
$$


The initial conditions and the boundary conditions on the exterior surface $\mathrm{S}$ and on the crack $\Sigma$ are respectively:

$$
\begin{array}{cc}
\mathrm{u}(\mathbf{x}, \mathrm{t}<0)=0, & \partial_{\mathrm{t}} \mathrm{u}(\mathbf{x}, \mathrm{t}<0)=0 \\
\left.\partial_{\mathrm{n}} \mathrm{u}\right|_{\mathrm{S}}=\Phi^{\mathrm{d}}, & \left.\partial_{\mathrm{n}} \mathrm{u}\right|_{\Sigma}=0
\end{array}
$$

We denote the solution by $u^{\varepsilon}$. As for the heat equation case, we consider additional assumptions on the boundary conditions such that the solution decreases at large time as

$$
\operatorname{Lim}_{t \rightarrow \infty} t^{2}\left|u^{\varepsilon}\right|=0, \quad \operatorname{Lim}_{t \rightarrow \infty} t^{2}\left|\partial_{t} u^{\varepsilon}\right|=0
$$

These conditions can be satisfied owing to Lions's theorem on the boundary controlability of dynamic systems (even for $\varepsilon=0$ ), stated in the so-called " Hilbert Uniqueness Method ». Following this method one can find a boundary loading condition for bringing a moving system at rest after some time $\mathrm{u}\left(\mathrm{x}, \mathrm{t}>\mathrm{t}_{1}\right)=0$, or, for imposing it any prescribed movement $\mathrm{u}(\mathbf{x}, \mathrm{t})$ satisfying (102) (Lions, 1986).

Equations (102) are necessary for obtaining the reciprocity functional for transient acoustics. As a matter of fact, the reciprocal relationship between the current field $u$ of a cracked body and an auxiliary $\mathrm{C}^{2}$ field $\mathrm{w}$ in the uncracked solid is written as follows (we omit the subscript $\varepsilon$ in $u^{\varepsilon}$ keeping in mind its asymptotic value $\varepsilon=0^{+}$, i.e. $\varepsilon>0, \varepsilon \rightarrow 0$ )

$$
\begin{gathered}
\int_{[0, \infty]} \int_{\Sigma}[[u]] \partial_{n} w d S d t=\int_{[0, \infty]} \int_{\Omega} u\left(\partial_{t} \partial_{t}-\Delta-\varepsilon \partial_{t}\right) w d V d t \\
+\int_{\Omega}\left(w \partial_{t} u-u \partial_{t} w+\varepsilon u w\right) \mid 0_{0}^{\infty} d V \\
+\int_{[0, \infty]} \int_{S}\left(u \partial_{n} w-w \partial_{n} u\right) d S d t
\end{gathered}
$$

The first term vanishes if $\mathrm{w}$ satisfies the adjoint equation :

$$
L^{*}[w] \equiv\left(\partial_{t} \partial_{t}-\Delta-\varepsilon \partial_{t}\right) w=0 \quad \text { in } \Omega \times[0, \infty[
$$

The term $\left(w \partial_{t} u-u \partial_{t} w+\varepsilon u w\right)$ vanishes at $t=0$. If it vanishes at large time $t-\rightarrow \infty$, then the second term in the r.h.s of (103) vanishes too. We obtain the dynamic reciprocity functional in the same form as in (89), with the boundary data pair $u=u^{d}, \partial_{n} u=\Phi d$

$$
\text { . } \int_{[0, \infty]} \int_{\Sigma}[[u]] \partial_{n} w d S d t=\int_{[0, \infty]} \int_{S}\left(u d \partial_{n} w-w \Phi d\right) d S d t
$$

Solution of the inverse problem. The determination of the normal $\mathbf{n}$ to the crack surface $\Sigma$ is simply done by using two plane waves of distinct propagation vectors $\mathbf{k}^{1}, \mathbf{k}^{2}$ parallel to the 
plane. Consider a wave $w^{k}(\mathbf{x}, t)=g(\mathbf{x} \cdot \mathbf{k}+t), \mathbf{k}=\mathbf{k}^{1}$ or $\mathbf{k}^{2}$ which do not vanish in $\Omega \times[0, T]$ and vanish in $\Omega \times\left[\mathrm{T}, \infty\left[\right.\right.$ and $\Omega \times[-\infty, 0]$ The gradient $\partial_{\mathrm{n}} \mathrm{w}=\mathbf{n} . \mathbf{k g}$ ' vanishes at any time if $\mathbf{n . k}=0$. Therefore the zeros of the reciprocity gap function $\mathbf{k} \rightarrow \mathrm{R} d(\mathbf{k})$, defined by the r.h.s. of (105) give a candidate for a propagation vector parallel to the crack plane. Two different zeros $\mathbf{k}^{1}$ and $\mathbf{k}^{2}$ of the reciprocity gap function $\mathbf{k} \rightarrow \mathrm{R}(\mathbf{k})$ determine the normal as $\mathbf{n}=\mathbf{k}^{1} \times \mathbf{k}^{2}$.

The position of the plane along $\mathrm{Ox}_{3}$ of the normal direction is defined by the equation $x_{3}-c=0$. To determine the constant $c$, we simply have to plot the function $b \rightarrow R^{d}(w(b)(x, t))$, and study the simple zero of this function with

$$
w^{(b)}(x, t)=\left(x_{3}-b\right)^{2}+(t-T / 2)^{2}
$$

which satisfies the adjoint equation (104) for $\varepsilon \rightarrow 0$. The above method of determining the crack plane by the reciprocity gap, using plane waves, are physically similar to the one used in Alves and Ha-Duong (1997) who studied the diffracted far-field by plane waves impinging the crack, called " analysing waves". When the analysing waves w are parallel to the crack plane, they are not diffracted by the crack, i.e they do not "see" the crack. One criterion for the non detection of crack by plane waves is the nullity of the reciprocity gap functional.

Let us show now how to choose the adjoint field for revealing the crack shape. Similar to the earlier exposition we consider the extension of the jump [[u]] to the whole crack plane by setting the jump extension equal to zero outside of the crack.

Let $\mathrm{D}\left(\mathrm{x}_{1}, \mathrm{x}_{2}, \mathrm{t}\right)=[[\mathrm{u}]], \mathrm{x}^{\prime}=\left(\mathrm{x}_{1}, \mathrm{x}_{2}, \mathrm{x}_{3}\right) \in \mathrm{P}^{3}, \mathrm{x}^{\prime}=\left(\mathrm{x}_{1}, \mathrm{x}_{2}\right) \in \mathrm{P}^{2}, \mathrm{~s}^{\prime}=\left(\mathrm{s}_{1}, \mathrm{~s}_{2}\right) \in \mathrm{P}^{2}$. Introduce the adjoint field depending on $\mathbf{s}^{\prime}$ and the real positive $\mathrm{q}>0$, with $\varepsilon=0^{+}$

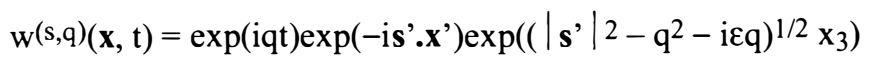

Let $\mathrm{H}\left(\mathbf{x}^{\prime} ; \mathrm{q}\right)=\int_{[0, \infty]}[[\mathrm{u}]] \exp (\mathrm{iqt}) \mathrm{dt}$ be the time Fourier transform of $[[\mathrm{u}]]$. The reciprocity gap can be written as

$$
\int_{\Pi} \mathrm{H}\left(\mathbf{x}^{\prime} ; \mathrm{q}\right) \exp \left(-\mathrm{i} \mathbf{s}^{\prime} . \mathbf{x}^{\prime}\right) \mathrm{dS}_{\mathrm{x}^{\prime}}=\left(\left|\mathbf{s}^{\prime}\right| 2-\mathrm{q}^{2}-\mathrm{i} 0^{+}\right)^{-1 / 2} \int_{[0, \infty]} \int_{\mathrm{S}}\left(\mathrm{u}^{\mathrm{d}} \partial_{\mathrm{n}} \mathrm{w}-\mathrm{w} \Phi^{\mathrm{d}}\right) \mathrm{dSdt}
$$

Therefore, the spatial Fourier transform of $\mathrm{H}\left(\mathbf{x}^{\prime} ; \mathrm{q}\right)$ is explicitly known from the boundary data $u^{d}, \Phi^{d}$ and the expression of the adjoint field $w \equiv w(s, q)$. The support of $H\left(x^{\prime} ; q\right)$ reveals the crack surface.

Proof of the inversion. The reason why $\varepsilon=0^{+}$has been introduced should now become clear. The function $\mathbf{s}^{\prime} \rightarrow\left(\left|\mathbf{s}^{\prime}\right|{ }^{2}-\mathrm{q}^{2}-\mathrm{i0}^{+}\right)^{-1 / 2}$ in the r.h.s. of $(108)$ is a distribution on the $\mathbf{s}^{\prime}$-plane, the extension of which to the complex $z^{\prime}$-planes, $\mathbf{z}^{\prime}=\left(\mathrm{s}_{1}+\mathrm{iv}_{1}, \mathrm{~s}_{2}+\mathrm{iv} \mathrm{v}_{2}\right)$ has no singularities in the real axes $s_{1}, s_{2}$. The r.h.s. of $(108)$ denoted by $F\left(s^{\prime}, q\right)$ is regular in the s'-plane, and can be analytically continued in the complex $\mathbf{z}$-planes as a function which has the same majoration of the " exponential type » as in (98). We conclude that for fixed $q>0$, the function $\mathbf{z}^{\prime} \rightarrow F\left(\mathbf{z}^{\prime}, q\right)$ is the spatial Fourier transform of the compact support distribution $\mathrm{H}\left(\mathbf{x}^{\prime}, \mathrm{q}\right)$ 


$$
H\left(\mathbf{x}^{\prime} ; q\right)=\left(1 / 4 \pi^{2}\right) \int_{R^{2}}\left(\left|\mathbf{s}^{\prime}\right| 2-q^{2}-i 0^{+}\right)^{-1 / 2} \exp \left(i \mathbf{s}^{\prime} \cdot \mathbf{x}^{\prime}\right)\left\{\int_{[0, \infty]} \int_{S}\left(\mathrm{u}^{\mathrm{d}} \partial_{\mathrm{n}} w-w \Phi^{d}\right) d S d t\right\} d s_{1} \mathrm{ds}_{2}
$$

The support of $\mathrm{H}\left(\mathbf{x}^{\prime}, \mathrm{q}\right)$ is the same as that of the jump [[u]].

\section{Concluding Remarks}

Inverse problems for defects and cracks identification are generally solved by optimization methods using two overdetermined sets of boundary data. Classically, one considers the observation equations or, more generally, the least square norm error between predicted and observed quantities. In this paper, we have considered new methods based on defects indicators, the constitutive law error, the analytical continuation (Cauchy problems) and the geometry bounds methods in statics as well as in dynamics.

By considering the variational form of the observation equation, in linear inverse problems, one deals with bilinear forms involving adjoint variables. The adjoint method is richer than the primal one, since it contains more informations about the hidden variables. This is particularly true for the observation equations deriving from the reciprocity gap functionals, considered as defects and cracks indicators. These new methods apply to nonlinear problems. By chosing appropriate adjoint fields, exact and explicit solutions can be obtained for a wide variety of inverse problems for identifying a planar crack in a $3 \mathrm{D}$ solid, ranging from elliptic to parabolic and hyperbolic equations.

\section{References}

Achenbach, J.D., Sotitopoulos, D.A., and Zhu, H. (1987). Characterisation of cracks from ultrasonic scattering data. J. Appl. Mech., 54, 754.

Alessandrini G, and Diaz Valenzuala A. (1996). Unique determination of multiple cracks by two measurements. SIAMJ. Control Optim. 34 (3), 913-921.

Alessandrini, G. (1993). Stable determination of a crack from boundary measurements. Proc. Roy. Society of Edinburgh, 123 (A), 497-516.

Alves C.J.S, and Ha-Duong T. (1999). Inverse scattering for elastic plane waves. Inverse Problems, 15, 91-97.

Alves C.J.S, and Ha-Duong T. (1997). On inverse scattering by screen. Inverse Problems 13, 1161-1176.

Andrieux S., and Ben Abda, A. (1992). Identification de fissures planes par une donnée de bord unique: un procédé direct de location et d'identification. C.R. Acad. Sci. Paris, 315 (I), 1323-1328.

Andrieux S., and Ben Abda, A. (1996). Identification of planar cracks by complete overdetermined data: inversion formulae. Inverse Problems, 12, 553-563.

Andrieux, S. Ben Abda, A., and Bui H. D. (1997) Sur l'identification des fissures planes via le concept d'écart à la réciprocité en élasticité. C.R. Acad. Sci.Paris, 324 (I), 1431-1438.

Andrieux, S. Ben Abda, A., and Bui H. D. (1999). Reciprocity principle and crack identification. Inverse Problems, 15, 59-65.

Arridge, S.R. (1999). Optical tomography in medical imaging. Inverse Problems, 15, R41-R93.

Bannour, T., Ben Abda, A., and Jaoua, M. (1997). A semi-explicit algorithmfor the reconstruction of 3D planar cracks. Inverse Problems 13, 899-917. 
Ben Abda, A., and Bui, H.D. (2003). Planar crack identification for the transient heat equation. J. Inv. Illposed Problems, 11 (1), 67-86.

Bojarski, N.N. (1981). Exact inverse scattering theory. Radio Sci., 16, 1025.

Bonnet, M., and Bui, H.D. (1989). On some inverse problems for determining volume defects by electric current using boundary integral equations approaches: an overview. Proc. $6^{\text {th }}$ Japan Nat. Symp. On Boundary Elements Methods, Tokyo, JASCOME Publ. 179.

Bryan, K, and Vogelius, M. (1992). A uniqueness result concerning the identification of a collection of cracks from finitely many elastostatic bundary measurements. SIAM J. Math. Anal. 23, 950-958.

Bui, H.D. (1993). Introduction aux problèmes inverses en mécanique des matériaux. Eyrolles, Paris; English translation "Inverse problems in the mechanics of materials: an introduction", CRC Press, Boca Raton (1994); Japanese translation, Shokabo, Tokyo (1994); Chinese translation, Harbin University Press (1995); Russian translation, Novosibirsk University Press (1996).

Bui, H.D., and Constantinescu, A. (2000). Spatial localization on the error of constitutive law for the identification of defects in elastic bodies. Arch. Mech., 52, 511-522.

Bui, H.D., Constantinescu, A., and Maigre, H. (1999). Diffraction acoustique inverse de fissure plane: solution explicite pour un solide borné. C.R. Acad. Sci. Paris, t. 327 (II), 971-976.

Calderon, A. (1980). On an inverse boundary problem. In Seminar on numerical analysis and application to continum physics, Rio de Janeiro, 65-73.

Constantinescu, A. (1994). Sur l'identification des modules élastiques. PhD Thesis, Ecole Polytechnique, Palaiseau.

Constantinescu, A. (1995). On the identification of elastic moduli from displacement-force boundary measurements. Int. J. Inverse Problems Engineering, 1, 293-315.

Dang Dinh Ang, Gorenflo, R., Le Khoi, V., and Dang, D.T. (2000). Moment theory and some inverse problems in potential theory and heat conduction. Springer, Berlin.

Friedman, A., and Vogelius, M. (1989). Determining cracks by boundary measurments. Indiana Univ. Math. J., 38 (3), 527-556.

Hadamard, J. (1952). Lectures on Cauchy's problem. Dover, New York.

Hasskell, N.A. (1984). Radiation pattern of surface waves from point sources in a multi-layered medium. Bulletin of seismological Society of America, 54, 377.

Ikehata, M. (1990). Inversion for the linearized problem for an inverse boundary value problem in elastic prospection. SIAM J. Appl. Math., 50 (6), 1635.

Isaacson, D., and Isaacson, E.L. (1989). Comment on Calderon's paper "On an inverse boundary value problem". Math. Compt., 52, 553.

Kitagawa, T., and Hosoda, Y. (1993). New approaches to the optimal regularisation. Inverse Problems in Engineering Mechanics, Tanaka M. and Bui H.D. (Eds), Springer Verlag, Berlin, 21.

Kohn, R.V., and Vogelius, M. (1984). Determining conductivity by measurements at boundary. Comm. Basic methods of tomography and inverse problems Pure Appl. Math. 37, 289.

Langenberg, K.J. (1987). Applied inverse problems for acoustic, electromagnetic wave scattering. In, Methods of tomography and inverse problemn, Sabatier P.C. (Ed.), Malvern Physics Ser., Adam Hilger, Bristol, Philadelphia.

Lattès, R., and Lions, J.L. (1967). Méthode de quasi-réversibilité et applications. Dunod, Paris.

Laurentiev, M.M. (1967).Some improperly posed problems of mathematical physics. Springer Verlag, Berlin.

Lions, J.L.(1986). Contrôlabilité exacte des systèmes distribués. C.R. Acad. Sci. Paris, 302 (II), 471-475. 
Lions, J.L. (1971). Optimal control of systems governed by partial derivative equations. Springer-Verlag, Berlin.

Lorentz, E., and Andrieux, S. (2003). Analysis of non-local models through energetic formulations. Int. J. Solids and Structures, 40, 2905-2936.

Nguyen, M.K., and Truong, T.T. (2002). On an integral transform and its inverse in nuclear imaging. Inverse Problems., 18 (1), 265-277.

Pontryagin, L.S., Boltianki, V., Gamkrelidze, R., and Michtchenko, E.F., (1974). Théorie mathématique des processus optimaux. Editions Mir, Moscou.

Sabatier, P.C. (1987). Methods of tomography and inverse problems. Malvern Phys. Ser., Adam Hilger, Bristol, Philadelphia.

Schwartz, L. (1978). Théorie des distribution. Hermann, Paris.

Stavroulakis, GE., (2000). Inverse and crack identification problems in engineering mechanics. Dordrecht, Kluwer.

Tikhonov, A., and Arsenine, V. (1976). Méthode de résolution de problèmes mal posés. Editions Mir, Moscou. English version : Methods for solving ill-posed problems, Nauka, Moscow.

Truong, T.T., Nguyen, M.K., Bui, H.D., and Daveau, C. (2002). Determination of the electronic density in a medium by an inverse method based on double-Compton scattering in transmission imaging. Proc. $4^{\text {th }}$ International Conf. on Inverse Problems in Engineering: Theory and Practice, Rio de Janeiro, May 2002

Weikl, W., Andra, H., and Schnack, E., (2001). An alternating iterative algorithm for the reconstruction of internal cracks in a three-dimensional solid body. Inverse Problems 17, 1957-1975. 